CERN-TH/95-216

SCIPP 95/52

MPI-PhT/95-103

NSF-ITP-96-62

TTP95-09用

hep-ph/9609331

\title{
APPROXIMATING THE RADIATIVELY CORRECTED HIGGS MASS IN THE MINIMAL SUPERSYMMETRIC MODEL
}

\author{
Howard E. Haber, ${ }^{1}$ Ralf Hempfling ${ }^{2}$ and André H. Hoang ${ }^{3}$ \\ ${ }^{1}$ CERN, TH-Division, CH-1211 Geneva 23, Switzerland \\ and \\ Santa Cruz Institute for Particle Physics \\ University of California, Santa Cruz, CA 95064 USA \\ ${ }^{2}$ Max-Planck-Institut für Physik, Werner-Heisenberg-Institut, \\ Föhringer Ring 6, D-80805 Munich, Germany \\ ${ }^{3}$ Institut für Theoretische Teilchenphysik, Universität Karlsruhe, \\ Kaiserstrasse 12, D-76128 Karlsruhe, Germany
}

\begin{abstract}
To obtain the most accurate predictions for the Higgs masses in the minimal supersymmetric model (MSSM), one should compute the full set of one-loop radiative corrections, resum the large logarithms to all orders, and add the dominant two-loop effects. A complete computation following this procedure yields a complex set of formulae which must be analyzed numerically. We discuss a very simple approximation scheme which includes the most important terms from each of the three components mentioned above. We estimate that the Higgs masses computed using our scheme lie within $2 \mathrm{GeV}$ of their theoretically predicted values over a very large fraction of MSSM parameter space.
\end{abstract}

CERN-TH/95-216

September, 1996

${ }^{*}$ The postscript file of this preprint, including figures, is available via anonymous ftp at ftp://www-ttp.physik.unikarlsruhe.de (129.13.102.139) as /ttp95-09/ttp95-09.ps or via www at http://www-ttp.physik.uni-karlsruhe.de/cgibin/preprints. 


\section{Introduction}

With the LEP-2 Collider now beginning operation, a detailed assessment of its discovery capabilities has recently been completed [1]. The search for the Higgs boson will play a central role in the LEP-2 program. A common rule of thumb states that for a center-of-mass energy $\sqrt{s}$, a Higgs boson with $m_{h^{0}} \lesssim \sqrt{s}-100 \mathrm{GeV}$ can be discovered (at the 5- $\sigma$ level), assuming a sufficient integrated luminosity. (Slightly higher masses can be ruled out at 95\% CL if no Higgs signal is seen.) This means that with $\sqrt{s}=192 \mathrm{GeV}$, one can expect to rule out or discover the Higgs boson if its mass is $\lesssim m_{Z}$.

In the Standard Model (SM), this Higgs mass discovery reach is not particularly impressive. Without any assumption about physics at higher energy scales (above, say, $1 \mathrm{TeV}$ ), Higgs masses up to about $700 \mathrm{GeV}$ are possible (within the context of a "weakly-coupled" scalar sector). On the other hand, if one assumes that no new physics (beyond the SM) enters up to the Planck scale, then one can deduce an upper bound of $m_{h^{0}} \lesssim 175 \mathrm{GeV}$ [2]. In this light, the LEP search is perhaps more significant. But, in the SM, there also exists a lower bound from stability arguments [3]. Under the assumption of no new physics up to the Planck scale, for $m_{t}=175 \mathrm{GeV}$, it follows that $m_{h^{0}} \gtrsim 125 \mathrm{GeV}$. Moreover, a Higgs boson discovery at LEP would imply that new physics must enter at an energy scale below $100 \mathrm{TeV}$. One of the leading candidates for such new physics is low-energy supersymmetry.

In the minimal supersymmetric extension of the Standard Model (MSSM), the Higgs sector [⿶] consists of two Higgs doublets of hypercharge \pm 1 . The scalar spectrum consists of two CP-even scalars, $h^{0}$ and $H^{0}$ (with $m_{h^{0}} \leq m_{H^{0}}$ ), a CP-odd scalar $A^{0}$ and a charged Higgs boson pair $H^{ \pm}$. Unlike the SM, the Higgs self-couplings are not independent parameters; but they are related to the electroweak gauge couplings. As a result, the Higgs mass spectrum is constrained. At tree-level, all Higgs masses and couplings depend on two parameters: $m_{A^{0}}$ and the ratio of Higgs vacuum expectation values, $\tan \beta$. The charged Higgs squared-mass is given by $\left(m_{H^{ \pm}}^{2}\right)_{0}=m_{A^{0}}^{2}+m_{W}^{2}$, while the CP-even neutral Higgs squared-masses and corresponding mixing angle $\alpha$ are obtained by diagonalizing the $2 \times 2$ mass matrix (in the hypercharge basis)

$$
\mathcal{M}_{0}^{2}=\left(\begin{array}{cc}
m_{A^{0}}^{2} \sin ^{2} \beta+m_{Z}^{2} \cos ^{2} \beta & -\left(m_{A^{0}}^{2}+m_{Z}^{2}\right) \sin \beta \cos \beta \\
-\left(m_{A^{0}}^{2}+m_{Z}^{2}\right) \sin \beta \cos \beta & m_{A^{0}}^{2} \cos ^{2} \beta+m_{Z}^{2} \sin ^{2} \beta
\end{array}\right),
$$

where the subscript 0 indicates tree-level quantities. One can then prove that $\left(m_{h^{0}}\right)_{0} \leq m_{Z}|\cos 2 \beta|$. If this tree-level inequality were reliable, then LEP-2 would have the mass reach either to discover the Higgs boson or rule out the MSSM! When radiative corrections are taken into account, the Higgs mass bound increases [5.66. The dominant contribution to this increase is a term of order $\Delta m_{h^{0}}^{2} \sim$ $3 g^{2} m_{t}^{4} \log \left(M_{\tilde{t}}^{2} / m_{t}^{2}\right) /\left(8 \pi^{2} m_{W}^{2}\right)$, which arises due to an incomplete cancellation of top quark and top squark loops (the cancellation would be complete in the limit of exact supersymmetry).

The radiative corrections to the Higgs mass have been computed by a number of techniques, and using a variety of approximations such as effective potential [6, 7, 8,9,9] and diagrammatic methods [5, 10, 11, 12, 13]. Complete one-loop diagrammatic computations of the MSSM Higgs masses have been presented by a number of groups [13]; the resulting expressions are quite complex, and depend on all the parameters of the MSSM. Moreover, as noted above, the largest contribution to the one-loop radiative corrections is enhanced by a factor of $m_{t}^{4}$ and grows logarithmically with the top squark mass. Thus, higher order radiative corrections can be non-negligible for a large top squark mass, in which case the large logarithms must be resummed. The renormalization group (RG) techniques for resumming the leading logarithms has been developed by a number of authors [14, 15, 16]. This procedure involves 
integrating a set of coupled partial differential equations. As a result, the numerical evaluation of the one-loop Higgs masses is time consuming and not very suitable for detailed phenomenological analyses. The primary goal of this paper is to present a simple algorithm that incorporates the effects of the RG-improvement and minimizes the size of the two-loop radiative corrections. We then can apply this algorithm to a suitable approximation to the full one-loop corrected Higgs masses.

We present a successive series of approximations to the one-loop corrected Higgs masses, of increasing complexity, each one reflecting finer details of the low-energy supersymmetric spectrum. Symbolically,

$$
\begin{aligned}
m_{H^{ \pm}}^{2} & =\left(m_{H^{ \pm}}^{2}\right)_{0}+\left(\Delta m_{H^{ \pm}}^{2}\right)_{1 \mathrm{LL}}+\left(\Delta m_{H^{ \pm}}^{2}\right)_{\text {mix }} \\
\mathcal{M}^{2} & =\mathcal{M}_{0}^{2}+\Delta \mathcal{M}_{1 \mathrm{LL}}^{2}+\Delta \mathcal{M}_{\mathrm{mix}}^{2}
\end{aligned}
$$

where the subscript 0 refers to the tree-level result, the subscript $1 L L$ refers to the one-loop leading logarithmic approximation to the full one-loop calculation, and the subscript mix refers to the contributions arising from $\widetilde{q}_{L}-\widetilde{q}_{R}$ mixing effects of the third generation squarks. The CP-even Higgs mass-squared eigenvalues are then given by

$$
m_{H^{0}, h^{0}}^{2}=\frac{1}{2}\left[\mathcal{M}_{11}^{2}+\mathcal{M}_{22}^{2} \pm \sqrt{\left[\mathcal{M}_{11}^{2}-\mathcal{M}_{22}^{2}\right]^{2}+4\left(\mathcal{M}_{12}^{2}\right)^{2}}\right]
$$

and the corresponding mixing angle, $\alpha$, is obtained from

$$
\begin{aligned}
\sin 2 \alpha & =\frac{2 \mathcal{M}_{12}^{2}}{\sqrt{\left[\mathcal{M}_{11}^{2}-\mathcal{M}_{22}^{2}\right]^{2}+4\left(\mathcal{M}_{12}^{2}\right)^{2}}}, \\
\cos 2 \alpha & =\frac{\mathcal{M}_{11}^{2}-\mathcal{M}_{22}^{2}}{\sqrt{\left[\mathcal{M}_{11}^{2}-\mathcal{M}_{22}^{2}\right]^{2}+4\left(\mathcal{M}_{12}^{2}\right)^{2}}} .
\end{aligned}
$$

In the first (and simplest) approximation, squark mixing effects are neglected and the supersymmetric spectrum is characterized by one scale, called $M_{\mathrm{SUSY}}$. We assume that $M_{\mathrm{SUSY}}$ is sufficiently large compared to $m_{Z}$ such that logarithmically enhanced terms at one-loop dominate over the nonlogarithmic terms. In this case, the full one-loop corrections (e.g., obtained by a diagrammatic technique) are well approximated by the one-loop leading logarithmic approximation. In this approximation, we neglect all squark mixing effects. Explicit formulae for $\Delta \mathcal{M}_{1 \mathrm{LL}}^{2}$ and $\left(\Delta m_{H^{ \pm}}^{2}\right)_{1 \mathrm{LL}}$ can be found in Appendix A. The second approximation incorporates squark mixing effects. These are likely to be significant only in the third generation squark sector. This approximation is parameterized by four supersymmetric parameters: $M_{\mathrm{SUSY}}$ (a common supersymmetric particle mass) and the third generation squark mixing parameters: $A_{t}, A_{b}$ and $\mu$. The corresponding formulae for $\Delta \mathcal{M}_{\text {mix }}^{2}$ and $\left(\Delta m_{H^{ \pm}}^{2}\right)_{\text {mix }}$ can be found in Appendix B.

The dominant contribution to the Higgs mass radiative corrections enters through the exchange of the third generation squarks. Thus, our third approximation treats this sector more precisely by accounting for non-degenerate top and bottom squark masses. This approximation is characterized by seven supersymmetric parameters - the three squark mixing parameters mentioned above, three soft-supersymmetry-breaking diagonal squark mass parameters, $M_{Q}, M_{U}$, and $M_{D}$, and a common

\footnotetext{
${ }^{a}$ If this condition does not hold, then the radiative corrections would constitute only a minor perturbation on the tree-level predictions.
} 
supersymmetry mass parameter $M_{\mathrm{SUSY}}$ which characterizes the masses of the first two generations of squarks, the sleptons, the charginos, and the neutralinos. A more precise set of formulae for $\Delta \mathcal{M}_{1 \mathrm{LL}}^{2}$ and $\Delta \mathcal{M}_{\text {mix }}^{2}$ incorporating the detailed squark and slepton mass spectrum, can be found in Appendix C. Note that setting $M_{Q}=M_{U}=M_{D}=M_{\mathrm{SUSY}}$ reduces this approximation to the previous one. Finally, our fourth approximation incorporates a non-trivial neutralino and chargino spectrum. This introduces two additional parameters, $M_{1}$ and $M_{2}$ which characterize the color singlet gaugino masses. The higgsino masses are determined by $\mu$, which already enters the analysis through the third generation squark mixing effects. The relevant correction terms to $\Delta \mathcal{M}_{1 \mathrm{LL}}^{2}$ incorporating a non-universal chargino and neutralino spectrum is given in Appendix D.

Given an approximation to the one-loop Higgs mass as described above we then develop a simple algorithm to incorporate the leading effects of RG-improvement. In Section 2, we demonstrate that the dominant higher order corrections can be absorbed in the expressions for the one-loop corrected Higgs masses by a suitable re-definition of $m_{t}$ (and $m_{b}$ ). Comparisons between the results of our analytic approximation and the results of the numerically integrated renormalization group equations (RGEs) demonstrate the domain of validity of our approximations. In Section 3, we include the effects of squark mixing. We are able to modify our analytic approximation in a simple way to incorporate the main squark mixing effects. Section 4 summarizes the results of this paper. In order to make the paper self contained, we collect in a series of appendices the necessary formulae required to implement our algorithm.

This paper was motivated in part by the Higgs Bosons working group of the 1995 LEP-2 Workshop [17], which examined in detail the phenomenology of the light CP-even and CP-odd Higgs bosons (in the MSSM). The radiative corrections to the light CP-even Higgs mass play a crucial role in determining the fraction of MSSM Higgs parameter space accessible to LEP-2 at its maximum energy of $\sqrt{s} \simeq 192 \mathrm{GeV}$. As a result, the graphs we present in this paper focus on the light CP-even

Higgs mass, although our formulae can also be used to compute the radiatively corrected masses of the heavy CP-even and charged Higgs bosons. While we were completing this work, Carena and collaborators published two papers in which analytic approximations to the radiatively corrected MSSM Higgs masses are also developed [9]. Their methods and emphasis are somewhat different from ours. Nevertheless, the final results are quite similar, and our numerical work (in cases where we have compared) typically agree to within $1 \mathrm{GeV}$ in the evaluation of Higgs masses. Some of the results of our work have been previously reported in Refs. [17] and [18], and our formulae have been employed in the review of Gunion, Stange, and Willenbrock in Ref. 19.

\section{$2 \quad$ RG-improved Higgs Masses-No Squark Mixing}

In this section, we examine the simplest case in which the supersymmetric spectrum is characterized by a single scale called $M_{\mathrm{SUSY}}$. We begin with the one-loop leading logarithmic expressions for the Higgs squared-masses given in Appendix A. Note that for $M_{\text {SUSY }} \gg m_{Z}$, the logarithmically enhanced terms appearing in the formulae of Appendix A can potentially spoil the perturbative expansion. In this case, it is necessary to perform a RG-improvement which resums the leading logs to all orders in perturbation theory. The resulting RG-improved perturbative expansion is better behaved and more reliable. The numerical effects of the RG-improvement can be significant for values of $M_{\mathrm{SUSY}}$ as low as $500 \mathrm{GeV}$. 
Numerical integration of the coupled RGEs is a straightforward but time-consuming process. Here, we develop a simple analytic formula that closely reproduces the result of the full numerical computation over the parameter regime of interest. Specifically, we are interested in values of $M_{\text {SUSY }}$ that lie between $200 \mathrm{GeV}$ and $2 \mathrm{TeV}$. For values of $M_{\mathrm{SUSY}}$ approaching $m_{Z}$, the leading logarithmic corrections are of the same size as non-leading corrections not included in the Appendix A formulae; however both are small corrections to the tree-level predictions. Moreover, in this regime, the effects of the RG-improvement are insignificant and can be neglected. For values of $M_{\text {SUSY }}$ above $2 \mathrm{TeV}$, the supersymmetry breaking scale is becoming unnaturally large (compared to the scale of electroweak symmetry breaking). In this section, we shall apply our technique to the one-loop leading logarithmic CP-even Higgs squared-mass matrix, $\mathcal{M}_{1 \mathrm{LL}}^{2} \equiv \mathcal{M}_{0}^{2}+\Delta \mathcal{M}_{1 \mathrm{LL}}^{2}$.

The matrix $\mathcal{M}_{1 \mathrm{LL}}^{2}$ depends explicitly on the top quark mass. But, which top-quark mass should one use? In a diagrammatic analysis, working in an on-shell scheme, one would use the pole mass. The analysis based on RG-running would naturally use the running mass, $m_{t}\left(m_{t}\right)$. The choice between the pole mass and $m_{t}\left(m_{t}\right)$ cannot be decided based on one-loop considerations alone. Since the dependence on $m_{t}$ enters only at one-loop, the distinction between various definitions of $m_{t}$ is a two-loop effect. We will return to this distinction later in this section. Dependence on $m_{b}$ will be considered below as well, although the numerical distinction among different $m_{b}$ choices is small.

We can now state a simple analytic formula that incorporates the dominant effects of the RG-improvement:

$$
\mathcal{M}_{1 \mathrm{RG}}^{2} \simeq \overline{\mathcal{M}^{2}} 1 \mathrm{LL} \equiv \mathcal{M}_{1 \mathrm{LL}}^{2}\left(m_{t}\left(\mu_{t}\right), m_{b}\left(\mu_{b}\right)\right), \quad \mu_{t} \equiv \sqrt{m_{t} M_{\mathrm{SUSY}}}, \quad \mu_{b} \equiv \sqrt{m_{Z} M_{\mathrm{SUSY}}} .
$$

That is, we assert that the numerically integrated RG-improved CP-even Higgs squared-mass matrix, $\mathcal{M}_{1 \mathrm{RG}}^{2}$, is well approximated by replacing all occurrences of $m_{t}$ and $m_{b}$ in $\mathcal{M}_{1 \mathrm{LL}}^{2}\left(m_{t}, m_{b}\right)$ by the corresponding running masses. [. Before justifying this assertion, we need formulae for $m_{b}(\mu)$ and $m_{t}(\mu)$. First, consider $m_{A^{0}}=\mathcal{O}\left(m_{Z}\right)$. In this case, at mass scales below $M_{\text {SUSY }}$, the effective theory of the Higgs sector is that of a non-supersymmetric two-Higgs-doublet model (2HDM). In this model, the quark mass is the product of the Higgs-quark Yukawa coupling $\left(h_{q}\right)$ and the appropriate Higgs vacuum expectation value:

$$
\begin{aligned}
m_{b}(\mu) & =\frac{1}{\sqrt{2}} h_{b}(\mu) v_{1}(\mu), \\
m_{t}(\mu) & =\frac{1}{\sqrt{2}} h_{t}(\mu) v_{2}(\mu),
\end{aligned}
$$

where we employ the normalization $v_{1}^{2}+v_{2}^{2}=4 m_{W}^{2} / g^{2}=(246 \mathrm{GeV})^{2}$. At scales $\mu \leq M_{\mathrm{SUSY}}$, we employ the one-loop non-supersymmetric RGEs of the 2HDM (see e.g., Ref. [20]) for $h_{b}, h_{t}$ and the vacuum expectation values. This yields

$$
\begin{aligned}
& \frac{\mathrm{d}}{\mathrm{d} \ln \mu^{2}} m_{b}^{2}=\frac{1}{64 \pi^{2}}\left[6 h_{b}^{2}+2 h_{t}^{2}-32 g_{s}^{2}+\frac{4}{3} g^{\prime 2}\right] m_{b}^{2}, \\
& \frac{\mathrm{d}}{\mathrm{d} \ln \mu^{2}} m_{t}^{2}=\frac{1}{64 \pi^{2}}\left[6 h_{t}^{2}+2 h_{b}^{2}-32 g_{s}^{2}-\frac{8}{3} g^{\prime 2}\right] m_{t}^{2} .
\end{aligned}
$$

For $m_{A^{0}}=\mathcal{O}\left(M_{\mathrm{SUSY}}\right)$, the effective theory of the Higgs sector at mass scales below $M_{\mathrm{SUSY}}$ is that of the one-Higgs doublet Standard Model. In this case, we define $m_{q}(\mu)=h_{q}^{\mathrm{SM}}(\mu) v(\mu) / \sqrt{2}$, where

\footnotetext{
${ }^{b}$ In this section, an overline above a quantity will indicate that the replacement of $m_{t}\left[m_{b}\right]$ by $m_{t}\left(\mu_{t}\right)\left[m_{b}\left(\mu_{b}\right)\right]$ has been made.
} 
$v\left(m_{Z}\right) \simeq 246 \mathrm{GeV}$ is the one-Higgs-doublet Standard Model vacuum expectation value. In this case eq. (2.7) is modified by replacing $6 h_{t}^{2}+2 h_{b}^{2}$ with $6\left(h_{t}^{\mathrm{SM}}\right)^{2}-6\left(h_{b}^{\mathrm{SM}}\right)^{2}$ in the RGE for $m_{t}^{2}$ (and interchange $b$ and $t$ to obtain the RGE for $m_{b}^{2}$ ).

To solve these equations, we also need the evolution equations of $g_{s}$, and $g^{\prime}$. But, an approximate solution is sufficient for our purposes. Since $g^{\prime}$ is small, we drop it. We do not neglect the $h_{b}$ dependence which may be significant if $\tan \beta$ is large. Then, we can iteratively solve eq. (2.7) to one loop by ignoring the $\mu$ dependence of the right hand side. We find

$$
m_{t}(\mu)=m_{t}\left(m_{t}\right) \times \begin{cases}1-\frac{1}{\pi}\left(\alpha_{s}-\frac{1}{16}\left(\alpha_{b}+3 \alpha_{t}\right)\right) \ln \left(\mu^{2} / m_{t}^{2}\right), & m_{A^{0}} \simeq \mathcal{O}\left(m_{Z}\right), \\ 1-\frac{1}{\pi}\left(\alpha_{s}-\frac{3}{16}\left(\alpha_{t}^{\mathrm{SM}}-\alpha_{b}^{\mathrm{SM}}\right)\right) \ln \left(\mu^{2} / m_{t}^{2}\right), & m_{A^{0}} \simeq \mathcal{O}\left(M_{\mathrm{SUSY}}\right)\end{cases}
$$

where $\alpha_{t} \equiv h_{t}^{2} / 4 \pi$, etc., and all coupling on the right hand side are evaluated at $m_{t}$. Similarly,

$$
m_{b}(\mu)=m_{b}\left(m_{Z}\right) \times \begin{cases}1-\frac{1}{\pi}\left(\alpha_{s}-\frac{1}{16}\left(\alpha_{t}+3 \alpha_{b}\right)\right) \ln \left(\mu^{2} / m_{Z}^{2}\right), & m_{A^{0}} \simeq \mathcal{O}\left(m_{Z}\right), \\ 1-\frac{1}{\pi}\left(\alpha_{s}-\frac{3}{16}\left(\alpha_{b}^{\mathrm{SM}}-\alpha_{t}^{\mathrm{SM}}\right)\right) \ln \left(\mu^{2} / m_{Z}^{2}\right), & m_{A^{0}} \simeq \mathcal{O}\left(M_{\mathrm{SUSY}}\right),\end{cases}
$$

For intermediate values of $m_{A^{0}}$, one may extrapolate the above formulae between the two regions indicated in the step function approximation. Using the results of eqs. (2.8) and (2.9) in eq. (2.5), and diagonalizing the squared-mass matrix of eq. (2.5) yields our approximation to the RG-improved one-loop neutral CP-even Higgs squared-masses.

Before justifying the above results, we exhibit a numerical comparison among various computations of the one-loop corrected light CP-even Higgs mass. First, we evaluate two expressions for the RG-unimproved one-loop Higgs mass - the one-loop leading log Higgs mass calculated from $\mathcal{M}_{1 \mathrm{LL}}^{2}$ and from a simplified version of $\mathcal{M}_{1 \mathrm{LL}}^{2}$ in which only the dominant terms proportional to $m_{t}^{4}$ are kept. In the latter case, we denote the CP-even Higgs squared-mass matrix by $\mathcal{M}_{1 \mathrm{LT}}^{2} \equiv \mathcal{M}_{0}^{2}+\Delta \mathcal{M}_{1 \mathrm{LT}}^{2}$, where

$$
\Delta \mathcal{M}_{1 \mathrm{LT}}^{2} \equiv \frac{3 g^{2} m_{t}^{4}}{8 \pi^{2} m_{W}^{2} \sin ^{2} \beta} \ln \left(M_{\mathrm{SUSY}}^{2} / m_{t}^{2}\right)\left(\begin{array}{cc}
0 & 0 \\
0 & 1
\end{array}\right)
$$

In many analyses of $\Delta \mathcal{M}_{1 \mathrm{LT}}^{2}$ and $\Delta \mathcal{M}_{1 \mathrm{LL}}^{2}$ that have appeared previously in the literature, the Higgs mass radiative corrections were evaluated with the pole mass, $m_{t}$. Some have argued that one should take $m_{t}$ to be the running mass evaluated at $m_{t}$, although to one-loop accuracy, the two choices cannot be distinguished. Nevertheless, because the leading radiative effect is proportional to $m_{t}^{4}$, the choice of $m_{t}$ in the one-loop formulae is numerically significant, and can lead to differences as large as $10 \mathrm{GeV}$ in the computed Higgs mass. At the end of this section, we justify the choice of using $m_{t}\left(m_{t}\right)$ as opposed to $m_{t}^{\text {pole }}$ (prior to RG-improvement) by invoking information from a two-loop analysis. Thus, our numerical results for the light CP-even Higgs mass before RG-improvement is significantly lower (when $M_{\text {SUSY }}$ is large) as compared to the original computations given in the literature, for fixed $m_{t}^{\text {pole }}$. In this paper, we have taken $m_{t}\left(m_{t}\right)=166.5 \mathrm{GeV}$ in all of our numerical work. We then apply our algorithm for RG-improvement by replacing $m_{t}$ and $m_{b}$ by their running masses evaluated at $\mu_{t}$ and $\mu_{b}$, respectively, as specified in eq. (2.5).

We now show examples for $m_{A^{0}}=1 \mathrm{TeV}$ and two choices of $\tan \beta$ in Figs. 1 and 2, and for $m_{A^{0}}=100 \mathrm{GeV}$ and $\tan \beta=20$ in Fig. 3. Each plot displays five predictions for $m_{h^{0}}$ based on the

${ }^{c}$ For $m_{A^{0}}=100 \mathrm{GeV}$ and $\tan \beta=1.5$, the resulting light Higgs mass lies below experimental Higgs mass bounds obtained by the LEP collaborations 21 . 
following methods for computing the Higgs squared-mass matrix: (i) $\mathcal{M}_{1 \mathrm{LT}}^{2}$; (ii) $\mathcal{M}_{1 \mathrm{LL}}^{2}$; (iii) $\overline{\mathcal{M}^{2}}{ }_{1 \mathrm{LT}}$ (iv) $\overline{\mathcal{M}}^{2}{ }_{1 \mathrm{LL}}$; and (v) $\mathcal{M}_{1 \mathrm{RG}}^{2}$ [see footnote below eq. (2.5)]. The following general features are noteworthy. First, we observe that over the region of $M_{\mathrm{SUSY}}$ shown, $\mathcal{M}_{1 \mathrm{RG}}^{2} \simeq \overline{\mathcal{M}}_{1 \mathrm{LL}}$. In fact, $m_{h^{0}}$ computed from $\overline{\mathcal{M}}^{2} 1 \mathrm{LL}$ is within $1 \mathrm{GeV}$ of the numerical RG-improved $m_{h^{0}}$ in all sensible regions of the parameter space $\left(1 \leq \tan \beta \leq m_{t} / m_{b}\right.$ and $\left.m_{t}, m_{A^{0}} \leq M_{\mathrm{SUSY}} \leq 2 \mathrm{TeV}\right)$. For values of $M_{\mathrm{SUSY}}>2 \mathrm{TeV}$, the Higgs masses obtained from $\overline{\mathcal{M}^{2}}$ 1LL begin to deviate from the numerically integrated RG-improved result. Second, the difference between $m_{h^{0}}$ computed from $\mathcal{M}_{1 \mathrm{LL}}^{2}$ and from $\mathcal{M}_{1 R G}^{2}$ is non-negligible for large values of $M_{\text {SUSY }}$; neglecting RG-improvement can lead to an overestimate of $m_{h^{0}}$ which in some areas of parameter space can be as much as $10 \mathrm{GeV}$. Finally, note that although the simplest approximation of $m_{h^{0}}$ based on $\mathcal{M}_{1 \mathrm{LT}}^{2}$ reflects the dominant radiative corrections, it yields the largest overestimate of the light Higgs boson mass.

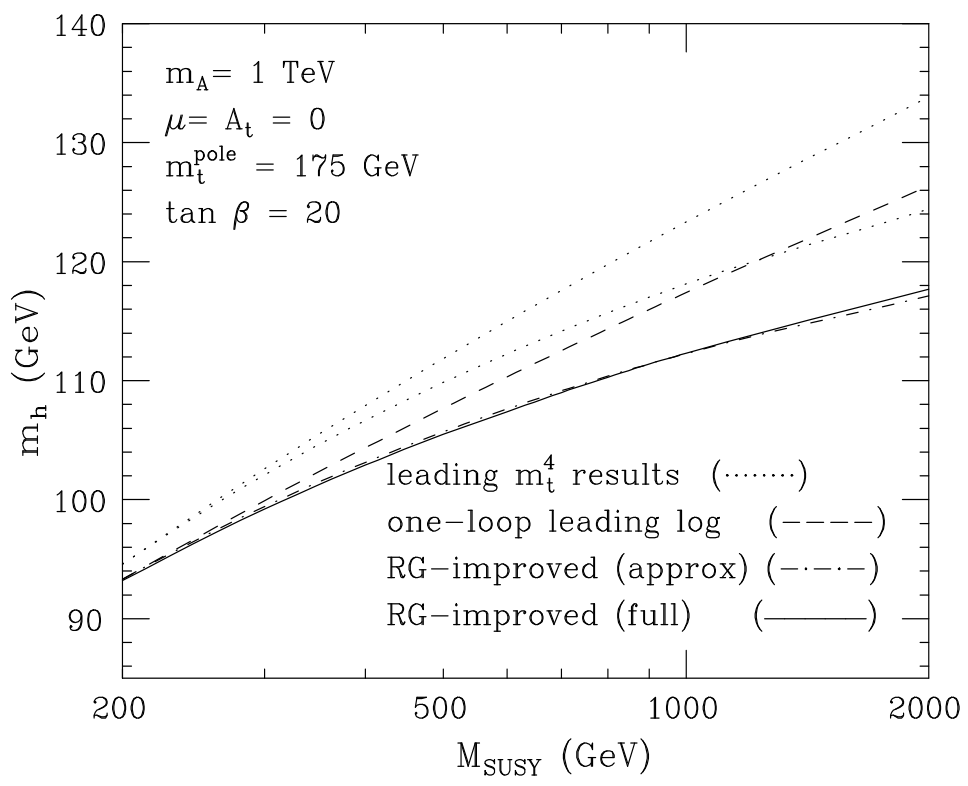

Fig. 1. The radiatively corrected light CP-even Higgs mass is plotted as a function of $M_{\mathrm{SUSY}}$ for $\tan \beta=20$ and $m_{A^{0}}=1 \mathrm{TeV}$. The one-loop leading logarithmic computation [dashed line] is compared with the RG-improved result which was obtained by numerical analysis [solid line] and by using the simple analytic result given in eq. (2.5) [dot-dashed line]. For comparison, the results obtained using the leading $m_{t}^{4}$ approximation of eq. (2.10) [higher dotted line], and its RG-improvement [lower dotted line] are also exhibited. $M_{\mathrm{SUSY}}$ characterizes the scale of supersymmetry breaking and can be regarded (approximately) as a common supersymmetric scalar mass; squark mixing effects are set to zero. The running top quark mass used in our numerical computations is $m_{t}\left(m_{t}\right)=166.5 \mathrm{GeV}$.

We now give a justification for the prescription proposed in eq. (2.5) for the radiatively corrected CP-even Higgs squared-mass matrix. Because the one-loop leading logarithmic correction proportional to $m_{t}^{4}$ is dominant, it is sufficient to consider the two-loop leading and next-to-leading $\log$ contributions proportional to $m_{t}^{4} \alpha_{s}$ and $m_{t}^{4} \alpha_{t}$. To simplify the presentation we will exhibit the 


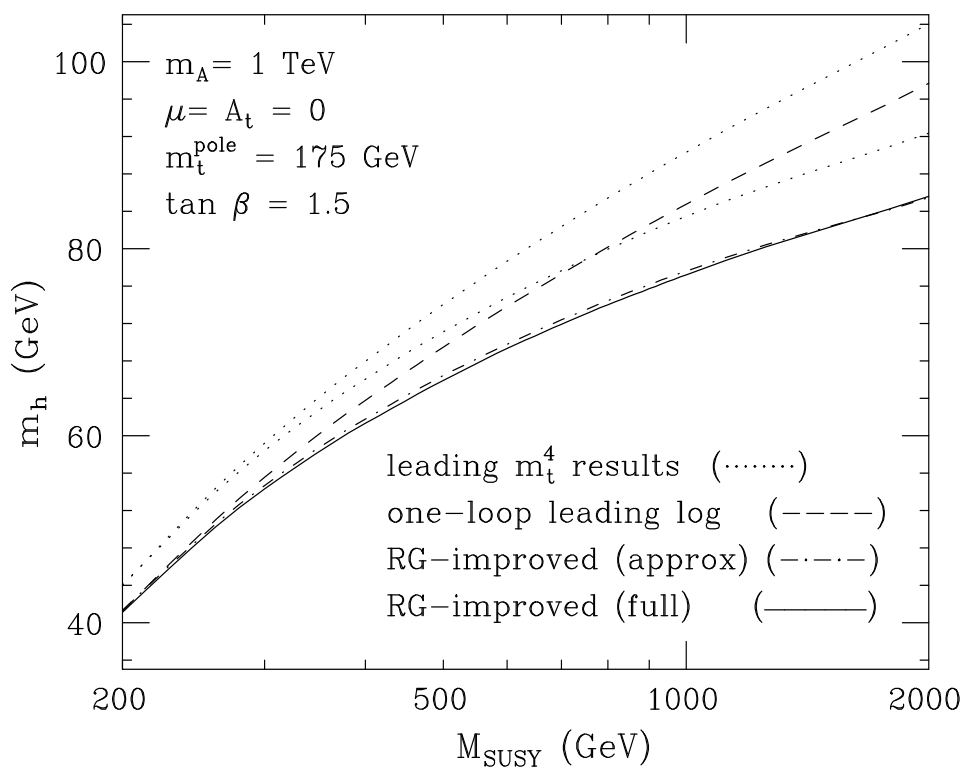

Fig. 2. The radiatively corrected light CP-even Higgs mass is plotted as a function of $M_{\mathrm{SUSY}}$ for $\tan \beta=1.5$ and $m_{A^{0}}=1 \mathrm{TeV}$. See the caption to Fig. 1. 1 .

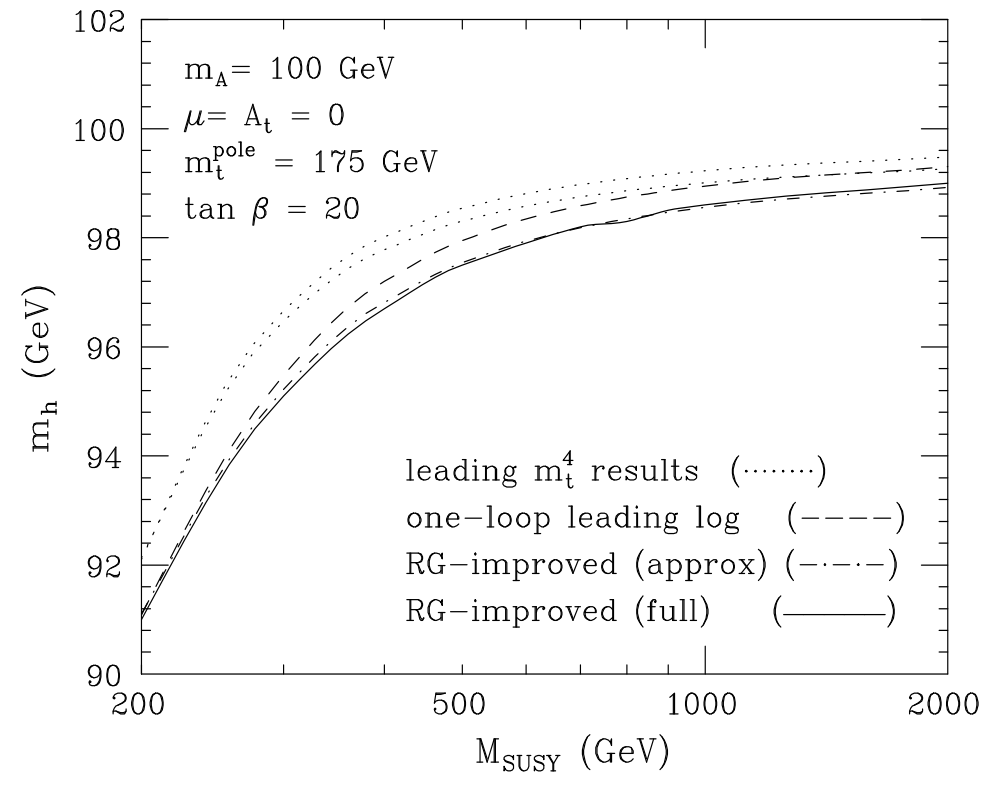

Fig. 3. The radiatively corrected light CP-even Higgs mass is plotted as a function of $M_{\mathrm{SUSY}}$ for $\tan \beta=20$ and $m_{A^{0}}=100 \mathrm{GeV}$. See the caption to Fig. 目. 
calculational steps for the case $m_{A^{0}} \sim \mathcal{O}\left(M_{\text {SUSY }}\right), m_{b}=0$, and $\beta=\pi / 2$. In this case, the effective Higgs sector at scales below $M_{\text {SUSY }}$ is the one-Higgs-doublet Standard Model, which is characterized by one Higgs self-coupling parameter $\lambda$. Supersymmetry fixes the value of $\lambda$ above the supersymmetry breaking scale, $M_{\text {SUSY }}$, to be

$$
\lambda\left(M_{\mathrm{SUSY}}\right)=\frac{1}{4}\left(g^{2}+g^{\prime 2}\right)\left(M_{\mathrm{SUSY}}\right) .
$$

The physical Higgs mass is determined at the scale $m_{Z}$ (assumed to be much less than $M_{\mathrm{SUSY}}$ ): $m_{h^{0}}=\lambda\left(m_{Z}\right) v^{2}\left(m_{Z}\right)$. Note that if supersymmetry were unbroken, then $\lambda$ would be fixed to the value given in eq. (2.11), which would imply that $m_{h^{0}}=m_{Z}$. This is the expected result for $\beta=\pi / 2$ and $m_{A^{0}}>m_{Z}$ [see eq. (1.1)]. In the case of broken supersymmetry, eq. (2.11) is taken as the boundary condition for the one-Higgs doublet Standard Model evolution of $\lambda$ from $M_{\text {SUSY }}$ down to $m_{Z}$. We work in the approximation $h_{b}=g=g^{\prime}=0$ (a more precise computation is not required here). In this approximation, we employ the following one-loop $\beta$-functions

$$
\begin{aligned}
& \beta_{\lambda} \equiv \frac{\mathrm{d}}{\mathrm{d} \ln \mu^{2}} \lambda=\frac{3}{8 \pi^{2}}\left[\lambda^{2}-h_{t}^{4}\right]-2 \lambda \gamma_{v}, \\
& \beta_{h_{t}^{2}} \equiv \frac{\mathrm{d}}{\mathrm{d} \ln \mu^{2}} h_{t}^{2}=\frac{1}{16 \pi^{2}}\left[\frac{9}{2} h_{t}^{2}-8 g_{s}^{2}\right] h_{t}^{2},
\end{aligned}
$$

where

$$
\gamma_{v} \equiv \frac{1}{v^{2}} \frac{\mathrm{d}}{\mathrm{d} \ln \mu^{2}} v^{2}=\frac{-3}{16 \pi^{2}} h_{t}^{2} .
$$

Solving iteratively the RGE for $\lambda$, and inserting the result in $m_{h^{0}}^{2}=\lambda\left(m_{Z}\right) v^{2}\left(m_{Z}\right)$, we end up with

$$
m_{h^{0}}^{2}=m_{Z}^{2}+\frac{3 g^{2} m_{t}^{4}\left(m_{t}\right)}{8 \pi^{2} m_{W}^{2}} \ln \left(\frac{M_{\mathrm{SUSY}}^{2}}{m_{t}^{2}}\right)\left[1+\left(\gamma_{v}+\frac{\beta_{h_{t}^{2}}}{h_{t}^{2}}\right) \ln \left(\frac{M_{\mathrm{SUSY}}^{2}}{m_{t}^{2}}\right)\right]
$$

where $h_{t} \equiv h_{t}\left(m_{t}\right)$ and $m_{t}\left(m_{t}\right)=h_{t} v\left(m_{t}\right) / \sqrt{2}$. Clearly, the term in brackets can be absorbed into the factor of $m_{t}^{4}$, thereby converting $m_{t}\left(m_{t}\right)$ into the running top quark mass evaluated at a new scale $\mu_{t}$. Comparison with eq. (2.8) shows that eq. (2.14) can be rewritten as

$$
m_{h^{0}}^{2}=m_{Z}^{2}+\frac{3 g^{2} m_{t}^{4}\left(\mu_{t}\right)}{8 \pi^{2} m_{W}^{2}} \ln \left(\frac{M_{\text {SUSY }}^{2}}{m_{t}^{2}}\right),
$$

where $\mu_{t} \equiv \sqrt{m_{t} M_{\mathrm{SUSY}}}$. Note that in the one-loop leading log analysis presented above, there is no distinction between $m_{t}\left(m_{t}\right)\left[m_{h^{0}}\left(m_{Z}\right)\right]$ and the top quark [Higgs boson] pole mass. Moreover, it is unclear at what scale to evaluate the factor of $m_{t}$ appearing in the argument of the logarithm in eq. (2.15). These ambiguities can be resolved by a two-loop analysis.

Ref. 12] used a diagrammatic technique to compute the leading two-loop contributions to the Higgs pole mass in terms of the top quark pole mass. In the approximation that $h_{b}=g=g^{\prime}=0$ and $M_{\mathrm{SUSY}} \gg m_{t}$ (and in the absence of squark mixing), the result of this computation can be written in the following form

$$
m_{h^{0}}^{2}=m_{Z}^{2}+\frac{3 g^{2}}{8 \pi^{2} m_{W}^{2}}\left(m_{t}^{\text {pole }}\right)^{4} \ln \left(\frac{M_{\text {SUSY }}^{2}}{m_{t}^{2}}\right)\left[1+\left(\gamma_{v}+\frac{\beta_{h_{t}^{2}}}{h_{t}^{2}}\right) \ln \left(\frac{M_{\text {SUSY }}^{2}}{m_{t}^{2}}\right)-\left(\frac{4 \alpha_{s}}{\pi}\right)+\frac{13}{8}\left(\frac{\alpha_{t}}{\pi}\right)\right] .
$$

\footnotetext{
${ }^{d}$ Note that in the approximation where $h_{b}=g=g^{\prime}=0$ and $\lambda$ is neglected compared with $h_{t}$, both $\lambda(\mu)$ and $v(\mu)$ do not run for $\mu \leq m_{t}$.

${ }^{e}$ In our two-loop analysis, we have defined $g^{2} / m_{W}^{2} \equiv 4 \sqrt{2} G_{\mu}$, where $G_{\mu}=1.16639 \times 10^{-5} \mathrm{GeV}^{-2}$ is the muon decay constant 22] and $m_{W}$ is the physical $W^{ \pm}$mass.
} 
In the same approximation as above, the top quark pole mass can be expressed (at one loop) in terms of the running top quark mass evaluated at $m_{t}$ [23,24,

$$
m_{t}^{\text {pole }}=m_{t}\left(m_{t}\right)\left[1+\frac{4}{3}\left(\frac{\alpha_{s}}{\pi}\right)-\frac{1}{2}\left(\frac{\alpha_{t}}{\pi}\right)\right]
$$

where $m_{t}\left(m_{t}\right)$ is defined below eq. (2.14). Inserting the above result into eq. (2.16) yields

$$
m_{h^{0}}^{2}=m_{Z}^{2}+\frac{3 g^{2}}{8 \pi^{2} m_{W}^{2}} m_{t}^{4}\left(m_{t}\right) \ln \left(\frac{M_{\mathrm{SUSY}}^{2}}{m_{t}^{2}}\right)\left[1+\left(\gamma_{v}+\frac{\beta_{h_{t}^{2}}}{h_{t}^{2}}\right) \ln \left(\frac{M_{\mathrm{SUSY}}^{2}}{m_{t}^{2}}\right)+\frac{4}{3}\left(\frac{\alpha_{s}}{\pi}\right)-\frac{3}{8}\left(\frac{\alpha_{t}}{\pi}\right)\right] .
$$

Given the Higgs mass computed to two-loop next-to-leading logarithmic accuracy, consider the error one makes by using eq. (2.14), where the two-loop next-to-leading logarithms are neglected. The size of the error depends on whether one uses the top quark pole mass or $m_{t}\left(m_{t}\right)$. By comparing eqs. (2.16) and (2.18), we see that the two-loop next-to-leading log term is a $10 \%$ correction if the top quark pole mass is used, whereas it is a $3 \%$ correction if $m_{t}\left(m_{t}\right)$ is used. This is our justification for using the running top-quark mass in our one-loop analysis.

One can also derive eq. (2.18) using the RG-techniques employed above. Only three modifications of our one-loop analysis are required. First, we must distinguish between the Higgs pole mass (denoted henceforth by $m_{h^{0}}$ with no argument) and the running Higgs mass evaluated at $m_{h^{0}}$. Using the results of Sirlin and Zucchini [25] (in the limit of $h_{b}=g=g^{\prime}=0$ and $\lambda \ll h_{t}$ ),

$$
m_{h^{0}}^{2}=\frac{4 m_{W}^{2} \lambda\left(m_{h^{0}}\right)}{g^{2}}\left[1+\frac{1}{8}\left(\frac{\alpha_{t}}{\pi}\right)\right] .
$$

Second, we need only the $h_{t}$ and $g_{s}$ dependent parts of the two loop contribution to $\beta_{\lambda}$. That is, $\beta_{\lambda}$ of eq. (2.12) is modified as follows [26]

$$
\beta_{\lambda} \longrightarrow \beta_{\lambda}+\frac{1}{\left(16 \pi^{2}\right)^{2}}\left[30 h_{t}^{6}-32 h_{t}^{4} g_{s}^{2}\right]
$$

Third, we must take into account a finite correction between $v^{2}\left(m_{t}\right)$ and $v^{2} \equiv 4 m_{W}^{2} / g^{2}$,

$$
v^{2}\left(m_{t}\right)=\frac{4 m_{W}^{2}}{g^{2}}\left[1-\frac{3}{8}\left(\frac{\alpha_{t}}{\pi}\right)\right] .
$$

This result can be deduced, for example, from Ref. [12]. Repeating the procedure leading to eq. (2.14) using the modifications indicated in eqs. (2.19)-(2.21), we easily reproduce eq. (2.18).

Finally, we can address the question of which top quark mass should appear in the argument of the logarithm. Using eq. (2.8) to convert the $m_{t}^{2}$ in the argument of the logarithm to $m_{t}^{2}\left(\mu_{t}\right)$, we find

$$
m_{h^{0}}^{2}=m_{Z}^{2}+\frac{3 g^{2}}{8 \pi^{2} m_{W}^{2}} m_{t}^{4}\left(\mu_{t}\right) \ln \left(\frac{M_{\mathrm{SUSY}}^{2}}{m_{t}^{2}\left(\mu_{t}\right)}\right)\left[1+\frac{1}{3}\left(\frac{\alpha_{s}}{\pi}\right)-\frac{3}{16}\left(\frac{\alpha_{t}}{\pi}\right)\right] .
$$

One can check that the sum of the terms in the brackets deviates from one by less than $1 \%$.

\footnotetext{
${ }^{f}$ We caution the reader that Ref. [24] defines $m_{t}\left(m_{t}\right)=2^{-3 / 4} G_{\mu}^{-1 / 2} h_{t}\left(m_{t}\right)$, which differs slightly from the definition of $m_{t}\left(m_{t}\right)$ used in our two-loop analysis.

${ }^{g}$ In Ref. [12] it is shown that the two-loop non-logarithmic piece is negligible.

${ }^{h}$ The numerical impact of the two-loop RGEs for the Higgs mass computation was examined in Ref. [15] and shown to be a few percent of the one-loop radiative corrections.
} 
Since the leading $m_{t}^{4}$ term provides the dominant source of the neutral Higgs mass radiative correction, it follows that our algorithm of replacing $m_{t}$ with $m_{t}\left(\mu_{t}\right)$ in the one-loop formula for $\mathcal{M}_{1 \mathrm{LL}}^{2}$ successfully reproduces the most important aspects of the RG-improvement while minimizing the effects of the non-leading-logarithmic two-loop effects, which are numerically small. Note that in the numerical work, we use eq. (2.8) to compute the running top-quark mass at the scale of $\mu_{t}$ in terms of $m_{t}\left(m_{t}\right)$. The latter quantity is expressed in terms of the top quark pole mass using the two-loop QCD corrections of Ref. [23] (approximately a 6\% effect) and the electroweak corrections of Ref. [24] (a $-0.4 \%$ correction for $m_{t}=175 \mathrm{GeV}$ and $m_{h^{0}} \sim m_{Z}$ ). . For $m_{t}^{\text {pole }}=175 \mathrm{GeV}$, this yields roughly $m_{t}\left(m_{t}\right)=166.5 \mathrm{GeV}$.

We may also apply our algorithm to the radiatively corrected charged Higgs mass. One can check that the twice-iterated two-loop leading log proportional to $m_{t}^{2} m_{b}^{2}$ is correctly reproduced. However, in contrast to the one-loop radiatively corrected neutral Higgs mass, there are no one-loop leading logarithmic corrections to $m_{H^{ \pm}}^{2}$ that are proportional to $m_{t}^{4}$. Thus, we expect that our charged Higgs mass approximation will not be quite as reliable as our neutral Higgs mass approximation.

\section{RG-Improved Higgs Masses-Squark Mixing Effects Included}

In the previous section, we focused on the radiative corrections under the assumption that one mass scale, $M_{\text {SUSY }}$ characterizes the supersymmetric masses. This is probably not a very realistic assumption. In this section, we focus on the effects arising from the mass splittings and $\widetilde{q}_{L}-\widetilde{q}_{R}$ mixing in the third generation squark sector. The latter can generate additional squared-mass shifts proportional to $m_{t}^{4}$ and thus can have a profound impact on the radiatively corrected Higgs masses. perform a RG-improvement of these effects, we follow the approach of Ref. [16]. First, we define our notation (we follow the conventions of Ref. [27]). In third family notation, the squark mass eigenstates are obtained by diagonalizing the following two $2 \times 2$ matrices. The top-squark squared-masses are eigenvalues of

$$
\left(\begin{array}{cc}
M_{Q}^{2}+m_{t}^{2}+t_{L} m_{Z}^{2} & m_{t} X_{t} \\
m_{t} X_{t} & M_{U}^{2}+m_{t}^{2}+t_{R} m_{Z}^{2}
\end{array}\right),
$$

where $X_{t} \equiv A_{t}-\mu \cot \beta, t_{L} \equiv\left(\frac{1}{2}-e_{t} \sin ^{2} \theta_{W}\right) \cos 2 \beta$ and $t_{R} \equiv e_{t} \sin ^{2} \theta_{W} \cos 2 \beta$, with $e_{t}=+2 / 3$. The bottom-squark squared-masses are eigenvalues of

$$
\left(\begin{array}{cc}
M_{Q}^{2}+m_{t}^{2}+b_{L} m_{Z}^{2} & m_{b} X_{b} \\
m_{b} X_{b} & M_{D}^{2}+m_{t}^{2}+b_{R} m_{Z}^{2}
\end{array}\right)
$$

where $X_{b} \equiv A_{b}-\mu \tan \beta, b_{L} \equiv\left(-\frac{1}{2}-e_{b} \sin ^{2} \theta_{W}\right) \cos 2 \beta$ and $b_{R} \equiv e_{b} \sin ^{2} \theta_{W} \cos 2 \beta$, with $e_{b}=-1 / 3$. $M_{Q}, M_{U}, M_{D}, A_{t}$, and $A_{b}$ are soft-supersymmetry-breaking parameters, and $\mu$ is the supersymmetric Higgs mass parameter. We treat the squark mixing perturbatively, assuming that the off-diagonal mixing terms are small compared to the diagonal terms. Two levels of approximation are considered. At the first level, we take $M_{Q}=M_{U}=M_{D}=M_{\mathrm{SUSY}}$, where $M_{\mathrm{SUSY}}$ is assumed to be large compared

\footnotetext{
${ }^{i}$ Since we are computing the radiatively corrected Higgs mass for arbitrary $m_{A^{0}}$ and $\tan \beta$, it is no longer appropriate to simply use eq. (2.17) for the top quark pole mass in terms of $m_{t}\left(m_{t}\right)$.

${ }^{j}$ The effects of mass splittings from other supersymmetric sectors to the radiatively corrected Higgs masses are quite small and will be neglected in the numerical analysis of this paper. However, in order to be complete, we present in Appendix D analytic expressions for the contributions from mass splittings in the chargino and neutralino sectors.
} 
to $m_{Z}$. Thus, the radiatively corrected Higgs mass is determined by $m_{A^{0}}, \tan \beta, M_{\mathrm{SUSY}}, A_{t}, A_{b}$, and $\mu$. At the second level, we allow $M_{Q}, M_{U}$, and $M_{D}$ to be arbitrary. However, as before, we assume that these soft-supersymmetry-breaking masses are large compared to $m_{Z}$.

At one-loop, the effect of the squark mixing is to introduce the shifts $\Delta \mathcal{M}_{\text {mix }}^{2}$ and $\left(\Delta m_{H^{ \pm}}^{2}\right)_{\text {mix }}$. For $M_{Q}=M_{U}=M_{D} \equiv M_{\mathrm{SUSY}}$, the relevant formulae are given in appendix B. For simplicity, we focus on this case in the remainder of this section. (For the case of arbitrary $M_{Q}, M_{U}$ and $M_{D}$, see Appendix C.) As in Section 2, we note the dependence of $\Delta \mathcal{M}_{\text {mix }}^{2}\left(m_{t}, m_{b}\right)$ on the top and bottom quark masses. If we evaluate $m_{t}$ and $m_{b}$ at a suitable scale, then it is possible to account for the dominant effects of the RG-improvement. Thus, for non-zero squark mixing, eq. (2.5) is generalized to:

$$
\mathcal{M}_{1 \mathrm{RG}}^{2} \simeq{\overline{\mathcal{M}^{2}}}_{1 \mathrm{LL}}+\Delta \overline{\mathcal{M}}_{\text {mix }} \equiv \mathcal{M}_{1 \mathrm{LL}}^{2}\left(m_{t}\left(\mu_{t}\right), m_{b}\left(\mu_{b}\right)\right)+\Delta \mathcal{M}_{\mathrm{mix}}^{2}\left(m_{t}\left(\mu_{\tilde{t}}\right), m_{b}\left(\mu_{\tilde{b}}\right)\right)
$$

where $\mu_{b}$ and $\mu_{t}$ are defined in eq. (2.5), and $\mu_{\tilde{q}}=M_{\text {SUSY }}(q=t, b)$. We have extended the overline notation of Section 2; note in particular that the scales at which one evaluates $m_{t}$ and $m_{b}$ are different in $\mathcal{M}_{1 \mathrm{LL}}^{2}$ and $\Delta \mathcal{M}_{\text {mix }}^{2}$, respectively. Intuitively, the squark mixing correction arises from integrating out heavy squarks that appear in one-loop corrections to Higgs scalar four-point functions. As a result, one should choose $\mu_{\tilde{q}}$ to coincide with the mass of the heaviest squark. A more detailed justification will be given below.

Following the discussion of Section 2, we compare the value of $m_{h^{0}}$ computed by different procedures. Prior to RG-improvement, we first compute $m_{h^{0}}$ by diagonalizing $\mathcal{M}_{1 \mathrm{LL}}^{2}+\Delta \mathcal{M}_{\mathrm{mix}}^{2}$. Next, we perform RG-improvement as in Ref. [16] by numerically integrating the RGEs for the Higgs self couplings starting from supersymmetric boundary conditions, and inserting the results into the diagonalized tree-level mass matrix. Finally, we compare the latter with $m_{h^{0}}$ computed by diagonalizing $\overline{\mathcal{M}}^{2} 1 \mathrm{LL}+\Delta \overline{\mathcal{M}}^{2}$ mix given by eq. 3.25$)$. These comparisons are exhibited in a series of figures. First, we plot $m_{h^{0}}$ vs. $X_{t} / M_{\mathrm{SUSY}}$ for $M_{\mathrm{SUSY}}=m_{A^{0}}=-\mu=1 \mathrm{TeV}$ for two choices of $\tan \beta$ in Fig. 1 [ $[\tan \beta=20]$ and Fig. $5[\tan \beta=1.5]$. Note that Fig. 4 is of particular interest, since it allows one to read off the maximal allowed value of $m_{h^{0}}$ for $M_{\mathrm{SUSY}} \leq 1 \mathrm{TeV}$. This maximal value occurs for $\left|X_{t}\right| \simeq 2.4 M_{\mathrm{SUSY}}$. The reader may worry that this value is too large in light of our perturbative treatment of the squark mixing. However, comparisons with exact diagrammatic computations confirm that these results are trustworthy at least up to the point where the curves reach their maxima. From a more practical point of view, such large values of the mixing are not very natural; they cause tremendous splitting in the top-squark mass eigenstates and are close to the region of parameter space where the $\mathrm{SU}(2) \times \mathrm{U}(1)$ breaking minimum of the scalar potential becomes unstable relative to color and/or electromagnetic breaking vacua [28].

In Figs. [1 and 5, $\mu=-1 \mathrm{TeV}$, i.e., as $X_{t} \equiv A_{t}-\mu \cot \beta$ varies, so does $A_{t}$. In fact, for $m_{A^{0}} \gg m_{Z}$, the dominant one-loop radiative corrections to $m_{h^{0}}^{2}$ depend only on $X_{t}$ and $M_{\mathrm{SUSY}}$ [see eq. (B.4)], so that for fixed $X_{t}$, the $\mu$ dependence of $m_{h^{0}}$ is quite weak. This is illustrated in Fig. 6. For values of $m_{A^{0}} \sim \mathcal{O}\left(m_{Z}\right)$, the $\mu$ dependence is slightly more pronounced (although less so for values of $\tan \beta \gg 1$ ) as illustrated in Fig. 7. We also display $m_{h^{0}}$ as a function of $M_{\text {SUSY }}$ for a number of different parameter choices in Fig. 8. In Fig. 9, we exhibit the $\tan \beta$ dependence of $m_{h^{0}}$ for two different choices of $X_{t}$. Again, we notice that our approximate formula [eq. (3.25)], which is depicted by the dot-dashed line, does remarkably well, and never differs from the numerically integrated RG-improved value (solid line) by more than $1.5 \mathrm{GeV}$ for $M_{\mathrm{SUSY}} \leq 2 \mathrm{TeV}$ and $\tan \beta \geq 1$. 


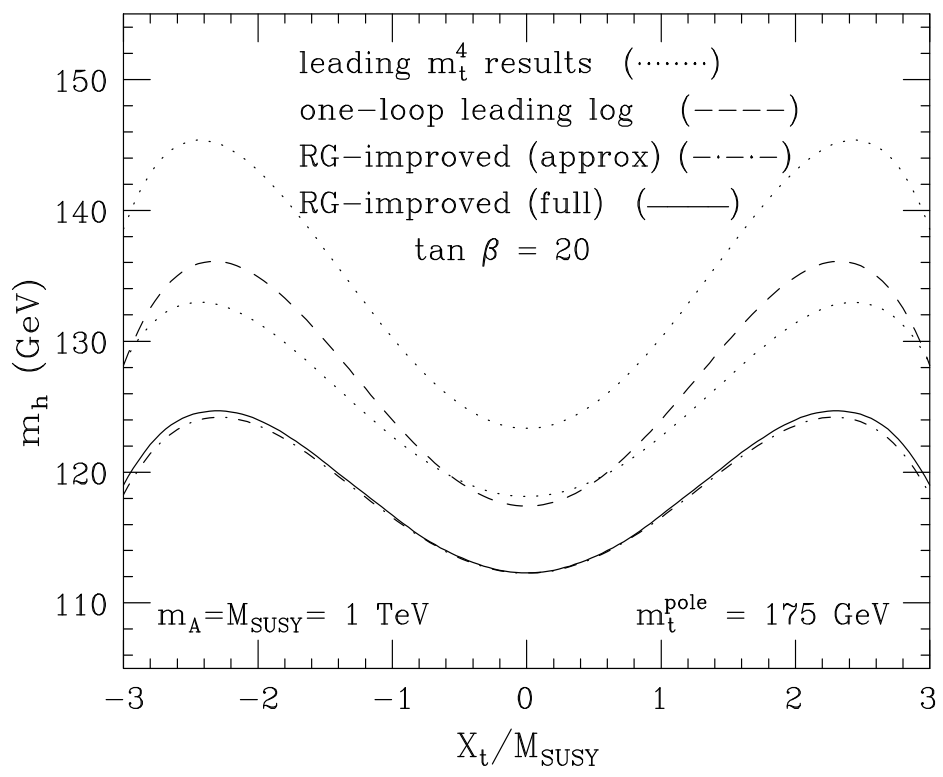

Fig. 4. The radiatively corrected light CP-even Higgs mass is plotted as a function of $X_{t} / M_{\mathrm{SUSY}}$, where $X_{t} \equiv A_{t}-\mu \cot \beta$, for $M_{\mathrm{SUSY}}=m_{A^{0}}=-\mu=1 \mathrm{TeV}$ and $\tan \beta=20$. See the caption to Fig. 1. 1 .

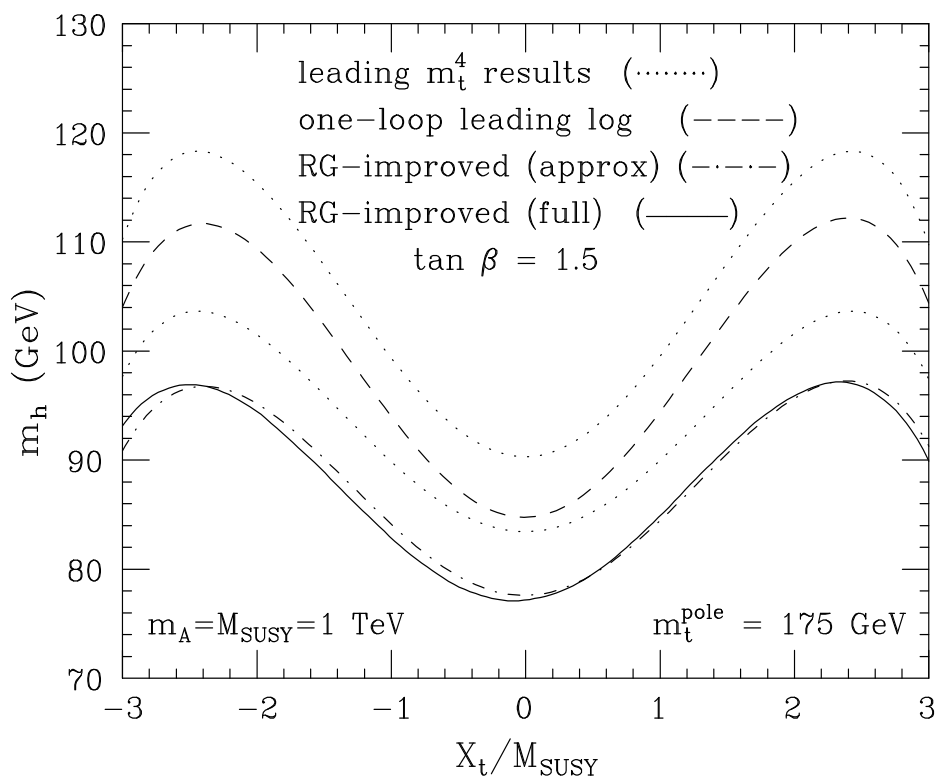

Fig. 5. The radiatively corrected light CP-even Higgs mass is plotted as a function of $X_{t} / M_{\mathrm{SUSY}}$, where $X_{t} \equiv A_{t}-\mu \cot \beta$, for $M_{\mathrm{SUSY}}=m_{A^{0}}=-\mu=1 \mathrm{TeV}$ and $\tan \beta=1.5$. See the caption to Fig. 1. 


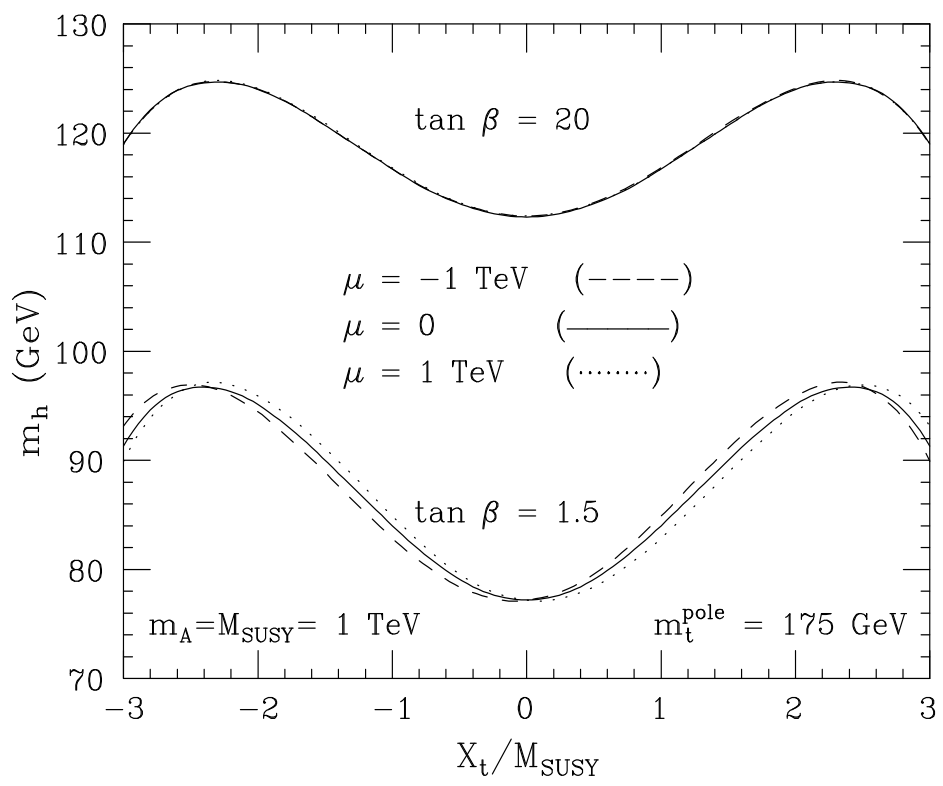

Fig. 6. The radiatively corrected, RG-improved light CP-even Higgs mass is plotted as a function of $X_{t} / M_{\mathrm{SUSY}}$, where $X_{t} \equiv A_{t}-\mu \cot \beta$, for $M_{\mathrm{SUSY}}=m_{A^{0}}=1 \mathrm{TeV}$ and two choices of $\tan \beta=1.5$ and 20 . Three values of $\mu$ are plotted in each case: $-1 \mathrm{TeV}$ [dashed], 0 [solid] and $1 \mathrm{TeV}$ [dotted]. Here, we have assumed that the diagonal squark squared-masses are degenerate: $M_{Q}=M_{U}=M_{D}=M_{\mathrm{SUSY}}$.

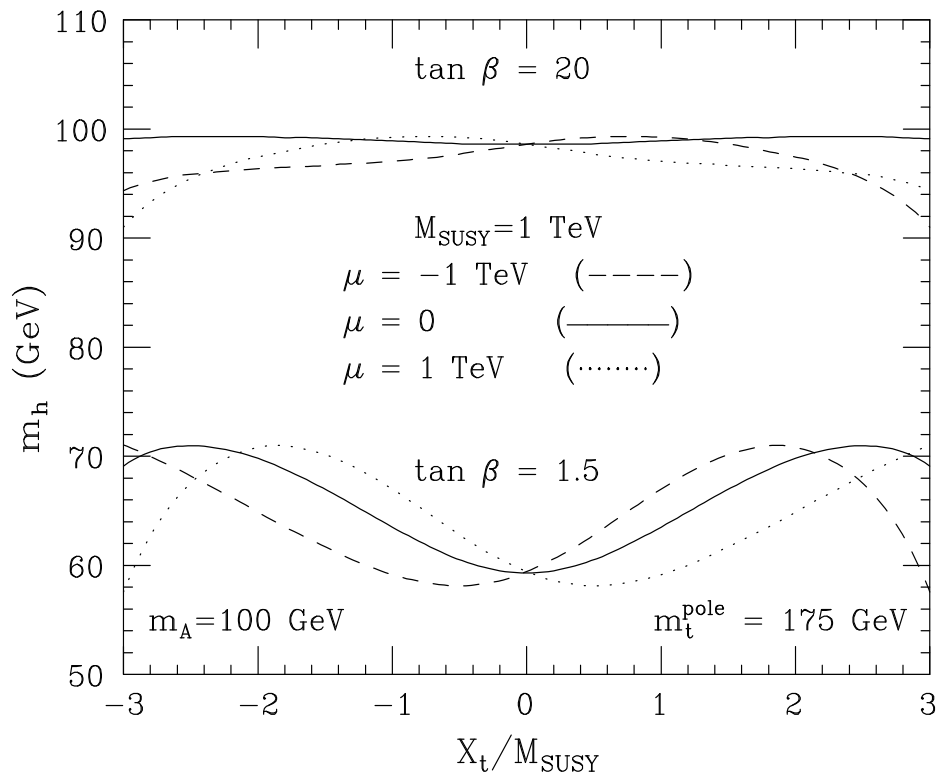

Fig. 7. The radiatively corrected, RG-improved light CP-even Higgs mass is plotted as a function of $X_{t} / M_{\mathrm{SUSY}}$ for $M_{\mathrm{SUSY}}=1 \mathrm{TeV}$ and $m_{A^{0}}=100 \mathrm{GeV}$. See the caption to Fig. 6 . 


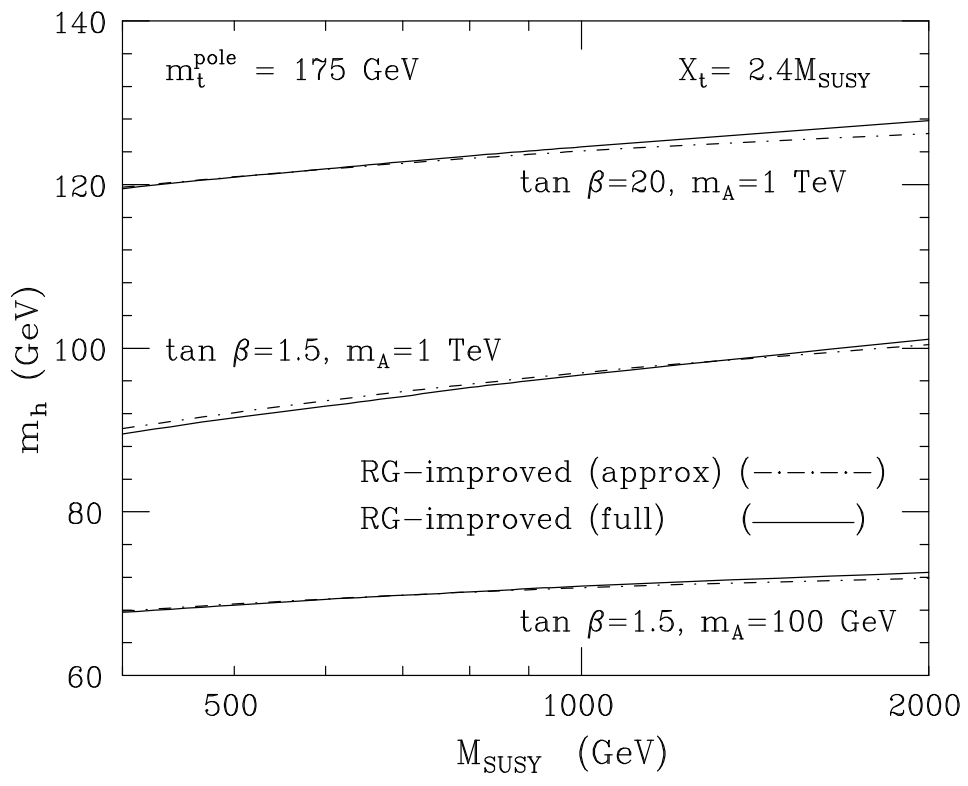

Fig. 8. The radiatively corrected, RG-improved light CP-even Higgs mass is plotted as a function of $M_{\mathrm{SUSY}}$ for $X_{t}=2.4 M_{\mathrm{SUSY}}$ for three choices of $\left(\tan \beta, m_{A^{0}}\right)=(20,1),(1.5,1)$, and $(1.5,0.1)$, where $m_{A^{0}}$ is specified in $\mathrm{TeV}$ units. The solid line depicts the numerically integrated result, and the dot-dashed line indicates the result obtained from eq. (3.25).

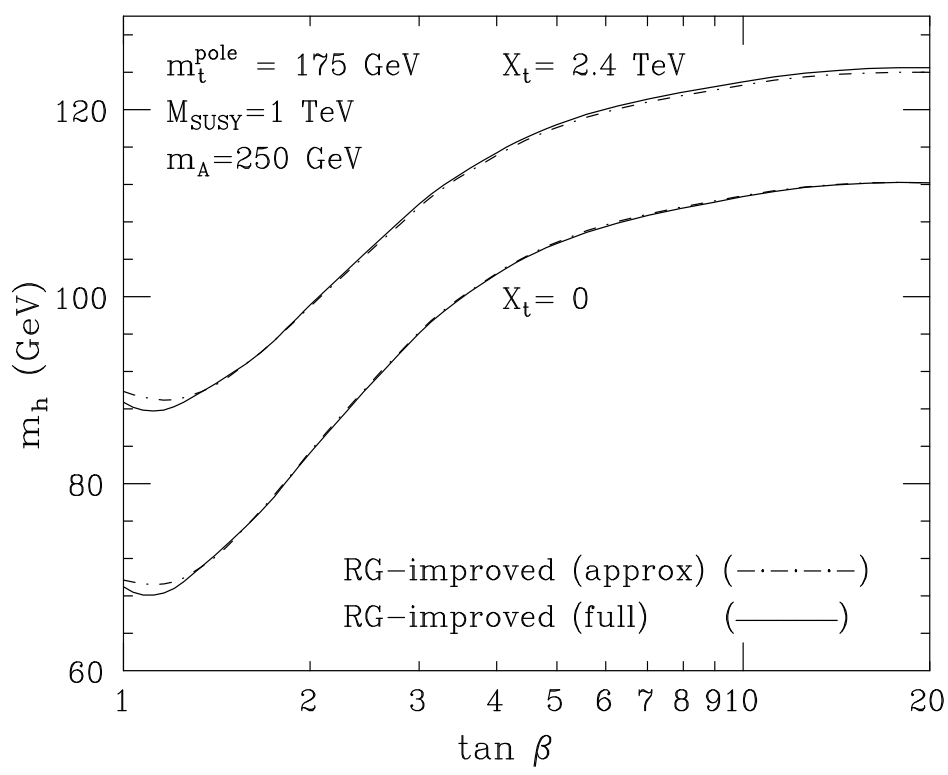

Fig. 9. The radiatively corrected, RG-improved light CP-even Higgs mass is plotted as a function of $\tan \beta$ for $M_{\mathrm{SUSY}}=1 \mathrm{TeV}$ and $m_{A^{0}}=250 \mathrm{GeV}$, for two choices of $X_{t}=0$ and $X_{t}=2.4 M_{\mathrm{SUSY}}$. See the caption to Fig. 8. 
Finally, we will present a brief derivation of eq. (3.25). As in Section 2, we simplify the analysis by taking $m_{A^{0}} \sim \mathcal{O}\left(M_{\mathrm{SUSY}}\right), m_{b}=0$, and $\beta=\pi / 2$. To incorporate the effects of top-squark mixing, eq. (2.11) is modified as follows

$$
\lambda\left(M_{\mathrm{SUSY}}\right)=\frac{1}{4}\left(g^{2}+g^{\prime 2}\right)+\frac{3 A_{t}^{2}}{8 \pi^{2} M_{\mathrm{SUSY}}^{2}}\left[1-\frac{A_{t}^{2}}{12 M_{\mathrm{SUSY}}^{2}}\right] h_{t}^{4}\left(M_{\mathrm{SUSY}}\right) .
$$

We may now repeat the steps of Section 2 to obtain:

$$
\left(\Delta m_{h^{0}}^{2}\right)_{\text {mix }}=\frac{3 g^{2} m_{t}^{4} A_{t}^{2}}{8 \pi^{2} m_{W}^{2} M_{\mathrm{SUSY}}^{2}}\left[1-\frac{A_{t}^{2}}{12 M_{\mathrm{SUSY}}^{2}}\right]\left[1+2\left(\gamma_{v}+\frac{\beta_{h_{t}^{2}}}{h_{t}^{2}}\right) \ln \left(\frac{M_{\mathrm{SUSY}}^{2}}{m_{t}^{2}}\right)\right] .
$$

Note the additional factor of 2 multiplying the logarithmic term as compared with eq. (2.14). Thus, by choosing the running top-quark mass at the scale $\mu_{\tilde{t}}=M_{\mathrm{SUSY}}$, one completely absorbs the logarithmic term,

$$
\left(\Delta m_{h^{0}}^{2}\right)_{\mathrm{mix}}=\frac{3 g^{2} A_{t}^{2}}{8 \pi^{2} m_{W}^{2} M_{\mathrm{SUSY}}^{2}}\left[1-\frac{A_{t}^{2}}{12 M_{\mathrm{SUSY}}^{2}}\right] m_{t}^{4}\left(\mu_{\tilde{t}}\right)
$$

\section{Discussion and Conclusions}

The masses and couplings of the Higgs bosons of the minimal supersymmetric model (MSSM) are calculable in terms of two parameters $\left(m_{A^{0}}\right.$ and $\left.\tan \beta\right)$ at tree-level. Including radiative corrections brings in a dependence on the MSSM particle spectrum, with dominant sensitivity to the top quark mass and the top squark masses and mixing parameters. Perhaps the most important prediction of the MSSM Higgs sector is the value of the lightest CP-even Higgs mass, $m_{h^{0}}$, which is predicted to be no greater than $m_{Z}|\cos 2 \beta|+\Delta m$, where $\Delta m$ depends on the size of the radiative corrections. This implies that a significant fraction of the MSSM Higgs parameter space is accessible to LEP-2. In this regard, an accurate computation of $m_{h^{0}}$ is crucial for a reliable assessment of the capabilities of LEP-2 (as a function of its center-of-mass energy). Presently known techniques allow one to compute $m_{h^{0}}$ with an accuracy of roughly $2 \mathrm{GeV}$. If the top squark masses are large $(\gtrsim 500 \mathrm{GeV})$, then it is crucial to include renormalization group (RG) improvement to obtain the accuracy just quoted. In this paper, we have presented a simple algorithm for including RG-improvement in the computation of Higgs masses. Our proposal can be applied to either the exact one-loop diagrammatic computation, or to approximations thereof. If we denote the one-loop CP-even Higgs squared-mass matrix by $\mathcal{M}^{2}\left(m_{t}, m_{b}, X_{t}, X_{b}\right)$, where we have indicated the dependence on the third generation quark mass and squark mixing parameters, then, the RG-improved squared-mass matrix is well approximated by

$$
\overline{\mathcal{M}^{2}} \equiv \mathcal{M}^{2}\left[m_{t}\left(\mu_{\tilde{t}}\right), m_{b}\left(\mu_{\tilde{b}}\right), X_{t}, X_{b}\right]-\mathcal{M}^{2}\left[m_{t}\left(\mu_{\tilde{t}}\right), m_{b}\left(\mu_{\tilde{b}}\right), 0,0\right]+\mathcal{M}^{2}\left[m_{t}\left(\mu_{t}\right), m_{b}\left(\mu_{b}\right), 0,0\right]
$$

This summarizes the results of eq. (2.5) and eq. (3.25). Note that eq. (4.29) can be applied even in the case where the effects of squark mixing are not explicitly separated out. As an example, consider the full diagrammatic one-loop computation, whose result we shall denote by $\mathcal{M}_{1 \mathrm{LC}}^{2}$. To carry out the

RG-improvement, one would subtract off the one-loop leading logarithmic pieces, $\mathcal{M}_{1 \mathrm{LL}}^{2}$, and replace it with its RG-improved value, $\mathcal{M}_{1 \mathrm{RG}}^{2}$. If we implement the RG-improvement using our analytic method described by eq. (4.29), we obtain

$$
\overline{\mathcal{M}}^{2}{ }_{1 \mathrm{LC}} \equiv \mathcal{M}_{1 \mathrm{LC}}^{2}+\overline{\mathcal{M}}^{2}{ }_{1 \mathrm{LL}}+\Delta \overline{\mathcal{M}}_{\text {mix }}-\mathcal{M}_{1 \mathrm{LL}}^{2}-\Delta \mathcal{M}_{\mathrm{mix}}^{2} .
$$


In cases that we have checked, we find that $\mathcal{M}_{1 \mathrm{LC}}^{2}-\mathcal{M}_{1 \mathrm{LL}}^{2}-\Delta \mathcal{M}_{\text {mix }}^{2}$ produces no more than about a $1 \mathrm{GeV}$ shift in the predicted Higgs masses. Thus, we expect that the numerical results obtained in this paper will shift by no more than $\sim 1 \mathrm{GeV}$ if non-leading logarithmic terms are included.

We find that if our algorithm is applied to the leading log one-loop corrections (given in Appendices A and C), plus the leading terms resulting from squark mixing (given in Appendices B and C), we reproduce the full (numerically integrated) RG-improved value of $m_{h^{0}}$, to within an accuracy of less than $2 \mathrm{GeV}$ (assuming that supersymmetric particle masses lie below $2 \mathrm{TeV}$ ). Although we have not focused our attention to the heavier Higgs states, we assert that our algorithm also yields accurate results for the mass of the heavier CP-even Higgs boson, $m_{H^{0}}$. Our approximation to the radiatively corrected charged Higgs mass is slightly less accurate only because the leading $m_{t}$ enhanced terms are not as dominant as in the neutral Higgs sector. Thus, the methods described in this paper provide a simple and powerful technique for obtaining an accurate determination of the radiatively corrected MSSM Higgs masses and CP-even mixing angle.

\section{Acknowledgments}

We gratefully acknowledge many fruitful interactions with Marcela Carena, Mariano Quiros, and Carlos Wagner. We also appreciate useful conversations with Damien Pierce and Fabio Zwirner. Two of us (HEH and RH) would like to thank the Institute for Theoretical Physics in Santa Barbara for their partial support under the National Science Foundation Grant No. PHY94-07194 during the final phase of this project. One of us $(\mathrm{HEH})$ is grateful for the hospitality of the Institut für Theoretische Teilchenphysik in Karlsruhe and the Max-Planck-Institut für Physik in Munich, where some of this work was accomplished. HEH is supported in part by the U.S. Department of Energy, and $\mathrm{AHH}$ is supported by the Graduiertenkolleg Elementarteilchenphysik, Karlsruhe.

\footnotetext{
${ }^{k}$ The approximation to the radiatively corrected charged Higgs mass can be improved by including sub-dominant terms not contained in the formulae given in this paper; see Ref. [11] for further details.
} 


\section{Appendix A: One-Loop Leading Logarithmic Corrections to the MSSM Higgs Masses}

We suppose that the supersymmetric particle spectrum is characterized by one mass scale, called $M_{\text {SUSY }}$ (assumed to be larger than $m_{t}$ ) Specifically, we assume that terms proportional to $\ln \left(M_{\text {SUSY }}^{2} / M_{X}^{2}\right)$, where $M_{X}$ stands for the mass of any supersymmetric particle, are much smaller than terms proportional to $\ln \left(M_{\text {SUSY }}^{2} / m_{Z}^{2}\right)$ and can be neglected. This assumption is made to keep the formulae that follow relatively simple. In fact, one can account for some spread in the supersymmetric mass spectrum by interpreting $M_{\text {SUSY }}$ appropriately depending on where it occurs in the formulae below. In this case additional correction terms arise, which are treated in Appendices $\mathrm{C}$ and D.

In the one-loop leading logarithmic approximation, the CP-even Higgs squared-mass matrix is given by:

$$
\mathcal{M}_{1 \mathrm{LL}}^{2}=\mathcal{M}_{0}^{2}+\Delta \mathcal{M}_{1 L L}^{2}
$$

where $\mathcal{M}_{0}^{2}$ is the tree level result given in eq. (1.1), and

$$
\begin{aligned}
\left(\Delta \mathcal{M}_{11}^{2}\right)_{1 \mathrm{LL}}= & \frac{g^{2} m_{Z}^{2} \cos ^{2} \beta}{96 \pi^{2} \cos ^{2} \theta_{W}}\left[P_{t} \ln \left(\frac{M_{\mathrm{SUSY}}^{2}}{m_{t}^{2}}\right)\right. \\
& +\left(12 N_{c} \frac{m_{b}^{4}}{m_{Z}^{4} \cos ^{4} \beta}-6 N_{c} \frac{m_{b}^{2}}{m_{Z}^{2} \cos ^{2} \beta}+P_{b}+P_{f}+P_{g}+P_{2 H}\right) \ln \left(\frac{M_{\mathrm{SUSY}}^{2}}{m_{Z}^{2}}\right) \\
& \left.+\Theta\left(m_{A^{0}}-m_{Z}\right)\left(P_{1 H}-P_{2 H}\right) \ln \left(\frac{m_{A^{0}}^{2}}{m_{Z}^{2}}\right)\right] \\
\left(\Delta \mathcal{M}_{22}^{2}\right)_{1 \mathrm{LL}}=\frac{g^{2} m_{Z}^{2} \sin ^{2} \beta}{96 \pi^{2} \cos ^{2} \theta_{W}}\left[\left(P_{b}+P_{f}+P_{g}+P_{2 H}\right) \ln \left(\frac{M_{\mathrm{SUSY}}^{2}}{m_{Z}^{2}}\right)\right. & +\left(12 N_{c} \frac{m_{t}^{4}}{m_{Z}^{4} \sin ^{4} \beta}-6 N_{c} \frac{m_{t}^{2}}{m_{Z}^{2} \sin ^{2} \beta}+P_{t}\right) \ln \left(\frac{M_{\mathrm{SUSY}}^{2}}{m_{t}^{2}}\right) \\
& \left.+\Theta\left(m_{A^{0}}-m_{Z}\right)\left(P_{1 H}-P_{2 H}\right) \ln \left(\frac{m_{A^{0}}^{2}}{m_{Z}^{2}}\right)+2 N_{c} \frac{m_{t}^{2}}{m_{Z}^{2} \sin ^{4} \beta}\right] \\
\left(\Delta \mathcal{M}_{12}^{2}\right)_{1 \mathrm{LL}=}= & \frac{g m_{Z}^{2} \sin _{3} \cos \beta}{96 \pi^{2} \cos ^{2} \theta_{W}}\left[\left(P_{t}-3 N_{c} \frac{m_{t}^{2}}{m_{Z}^{2} \sin ^{2} \beta}\right) \ln \left(\frac{M_{\mathrm{SUSY}}^{2}}{m_{t}^{2}}\right)\right. \\
& +\left(P_{b}-3 N_{c} \frac{m_{b}^{2}}{m_{Z}^{2} \cos ^{2} \beta}+P_{f}+P_{g}^{\prime}+P_{2 H}^{\prime}\right) \ln \left(\frac{M_{\mathrm{SUSY}}^{2}}{m_{Z}^{2}}\right) \\
& \left.-\Theta\left(m_{A^{0}}-m_{Z}\right)\left(P_{1 H}+P_{2 H}^{\prime}\right) \ln \left(\frac{m_{A^{0}}^{2}}{m_{Z}^{2}}\right)\right] \cdot
\end{aligned}
$$

\footnotetext{
${ }^{l}$ For a widely spread supersymmetric particle spectrum where this approximation is inappropriate, these corrections are listed in Appendices B and C (squarks and sleptons) and D (charginos and neutralinos).
} 
In eq. (A.2), the constants $P_{i}$ are defined as

$$
\begin{aligned}
P_{t} & \equiv N_{c}\left(1-4 e_{t} \sin ^{2} \theta_{W}+8 e_{t}^{2} \sin ^{4} \theta_{W}\right), \\
P_{b} & \equiv N_{c}\left(1+4 e_{b} \sin ^{2} \theta_{W}+8 e_{b}^{2} \sin ^{4} \theta_{W}\right), \\
P_{f} & \equiv N_{c}\left(N_{g}-1\right)\left[2-4 \sin ^{2} \theta_{W}+8\left(e_{t}^{2}+e_{b}^{2}\right) \sin ^{4} \theta_{W}\right]+N_{g}\left[2-4 \sin ^{2} \theta_{W}+8 \sin ^{4} \theta_{W}\right], \\
P_{g} & \equiv-44+106 \sin ^{2} \theta_{W}-62 \sin ^{4} \theta_{W}, \\
P_{g}^{\prime} & \equiv 10+34 \sin ^{2} \theta_{W}-26 \sin ^{4} \theta_{W}, \\
P_{2 H} & \equiv-10+2 \sin ^{2} \theta_{W}-2 \sin ^{4} \theta_{W}, \\
P_{2 H}^{\prime} & \equiv 8-22 \sin ^{2} \theta_{W}+10 \sin ^{4} \theta_{W}, \\
P_{1 H} & \equiv-9 \cos ^{4} 2 \beta+\left(1-2 \sin ^{2} \theta_{W}+2 \sin ^{4} \theta_{W}\right) \cos ^{2} 2 \beta,
\end{aligned}
$$

with the number of colors, $N_{c}=3$, the number of generations, $N_{g}=3$, and the quark charges given by $e_{t} \equiv 2 / 3$ and $e_{b} \equiv-1 / 3$. The origin of the various terms above can be found in Ref. [16]. The most important contribution to the one-loop radiative corrections above arises from the term in $\Delta \mathcal{M}_{22}^{2}$ that is proportional to $m_{t}^{4}$. If one discards all other one-loop radiative corrections, then one obtains $\Delta \mathcal{M}_{1 \mathrm{LT}}^{2}$ given in eq. (2.10).

In principle, the two cases $m_{A^{0}} \lesssim \mathcal{O}\left(m_{Z}\right)$ and $m_{A^{0}} \gg m_{Z}$ must be treated separately. However, by including the terms proportional to $\ln \left(m_{A^{0}}^{2} / m_{Z}^{2}\right)$ in eq. A.2), $m$ we interpolate between the small and large $m_{A^{0}}$ cases. This yields the correct leading log expression for $m_{h^{0}}$. The large $m_{A^{0}}$ analysis is more conveniently done in a basis where $h^{0}$ and $H^{0}$ are approximate eigenstates. If $\mathcal{R}$ is the rotation matrix which rotates a 2 -vector counterclockwise by angle $\beta$, then $\mathcal{R}^{-1} \mathcal{M}^{2} \mathcal{R}$ yields

$$
\left(\begin{array}{cc}
m_{h^{0}}^{2} & m_{h H}^{2} \\
m_{h H}^{2} & m_{H^{0}}^{2}
\end{array}\right) \simeq\left(\begin{array}{cc}
\mathcal{M}_{11}^{2} \cos ^{2} \beta+\mathcal{M}_{22}^{2} \sin ^{2} \beta+\mathcal{M}_{12}^{2} \sin 2 \beta & \frac{1}{2}\left(\mathcal{M}_{22}^{2}-\mathcal{M}_{11}^{2}\right) \sin 2 \beta+\mathcal{M}_{12}^{2} \cos 2 \beta \\
\frac{1}{2}\left(\mathcal{M}_{22}^{2}-\mathcal{M}_{11}^{2}\right) \sin 2 \beta+\mathcal{M}_{12}^{2} \cos 2 \beta & \mathcal{M}_{11}^{2} \sin ^{2} \beta+\mathcal{M}_{22}^{2} \cos ^{2} \beta-\mathcal{M}_{12}^{2} \sin 2 \beta
\end{array}\right),
$$

where the identification of the diagonal elements above follows from the fact that $m_{h H}^{2}=\mathcal{O}\left(m_{Z}^{2}\right)$ in the limit of $m_{A^{0}} \gg m_{Z}$. Using the above results, it follows that for $m_{A^{0}} \gg m_{Z}$,

$$
\begin{aligned}
\left(m_{h^{0}}^{2}\right)_{1 \mathrm{LL}}= & m_{Z}^{2} \cos ^{2} 2 \beta+\frac{g^{2} m_{Z}^{2}}{96 \pi^{2} \cos ^{2} \theta_{W}}\left\{\left[12 N_{c} \frac{m_{b}^{4}}{m_{Z}^{4}}-6 N_{c} \frac{m_{b}^{2}}{m_{Z}^{2}} \cos 2 \beta+P_{f} \cos ^{2} 2 \beta\right.\right. \\
& \left.+\left(P_{g}+P_{2 H}\right)\left(\sin ^{4} \beta+\cos ^{4} \beta\right)-2\left(P_{g}^{\prime}+P_{2 H}^{\prime}\right) \sin ^{2} \beta \cos ^{2} \beta\right] \ln \left(\frac{M_{\mathrm{SUSY}}^{2}}{m_{Z}^{2}}\right) \\
& +\left[12 N_{c} \frac{m_{t}^{4}}{m_{Z}^{4}}+6 N_{c} \frac{m_{t}^{2}}{m_{Z}^{2}} \cos 2 \beta+P_{t} \cos ^{2} 2 \beta\right] \ln \left(\frac{M_{\mathrm{SUSY}}^{2}}{m_{t}^{2}}\right) \\
& \left.-\left[P_{2 H}\left(\cos ^{4} \beta+\sin ^{4} \beta\right)-2 P_{2 H}^{\prime} \cos ^{2} \beta \sin ^{2} \beta-P_{1 H}\right] \ln \left(\frac{m_{A^{0}}^{2}}{m_{Z}^{2}}\right)\right\} \\
\left(m_{H^{0}}^{2}\right)_{1 \mathrm{LL}}= & m_{A^{0}}^{2}+m_{Z}^{2} \sin ^{2} 2 \beta+\mathcal{O}\left(g^{2} m_{Z}^{2}\right), \\
\left(m_{h H}^{2}\right)_{1 \mathrm{LL}}= & -m_{Z}^{2} \sin 2 \beta \cos 2 \beta+\mathcal{O}\left(g^{2} m_{Z}^{2}\right) .
\end{aligned}
$$

In order to compare the results of our analytic approximation to the RG-improved Higgs squared-mass [eq. (2.5)], one must integrate the Higgs self-couplings of the two-Higgs-doublet model from $M_{\mathrm{SUSY}}$

\footnotetext{
${ }^{m}$ Strictly speaking, these terms are meaningful for $m_{Z} \ll m_{A^{0}} \leq M_{\text {SUSY }}$. The step function in eq. (A.2) implies that these terms should not be included if $m_{A^{0}} \leq m_{Z}$.
} 
down to $m_{A^{0}}$, match onto the one-Higgs-doublet model, and finally evolve the Standard Model Higgs self-coupling from $m_{A^{0}}$ down to $m_{Z}$. However, we note that the contribution of the terms proportional to $\ln \left(m_{A^{0}}^{2} / m_{Z}^{2}\right)$ are quite small, leading to a mass shift in $m_{h^{0}}$ of no more than $1 \mathrm{GeV}$ (for $m_{A^{0}} \leq$ $1 \mathrm{TeV}$ ). As a result, we simplified the analysis in generating the graphs shown in Section 2 and 3 by omitting the $m_{A^{0}}$ dependence in the radiative corrections. ${ }^{n}$ That is, we omitted all terms proportional to $\ln \left(m_{A^{0}}^{2} / m_{Z}^{2}\right)$ that appear in the formulae above. We then compared the analytic approximation so obtained with the two-Higgs-doublet model numerically integrated from $M_{\text {SUSY }}$ down to $m_{Z}$, with no matching onto the one-Higgs-doublet model as described above. In this approximation, the dependence of the Higgs masses on $m_{A^{0}}$ enters only through the tree-level expressions. The impact of the $m_{A^{0}}$ dependence of the radiative corrections will be discussed briefly at the end of Appendix C.

Although the one-loop leading log terms in $m_{h^{0}}^{2}$ are correctly reproduced by using the results given in eqs. (A.2)-A.5, the analogous terms in $m_{H^{0}}^{2}$ obtained by the above procedure do not catch all terms proportional to $\ln \left(m_{A^{0}}^{2} / m_{Z}^{2}\right)$. The reason is related to the fact that below the scale $m_{A^{0}}$, the effective low-energy Higgs sector consists of just one scalar, $h^{0}$. Thus, for example, for $m_{A^{0}}=M_{\text {SUSY }}$ one would find $m_{H^{0}}^{2}=m_{A^{0}}^{2}+m_{Z}^{2} \sin ^{2} 2 \beta$, where the parameters on the right hand side should be considered as running parameters evaluated at the scale $m_{A^{0}}$ (and similarly for $m_{h H}^{2}=$ $\left.-m_{Z}^{2} \sin 2 \beta \cos 2 \beta\right)$. Then, the correct leading log pieces in the unspecified $\mathcal{O}\left(g^{2} m_{Z}^{2}\right)$ terms in eq. (A.5) would be obtained once the running parameters were expressed in terms of physical parameters. We shall not provide explicit expressions for the resulting $\mathcal{O}\left(g^{2} m_{Z}^{2}\right)$ terms, since these corrections $\rho$ yield only a small fractional shift to $m_{H^{0}}^{2}$. One subtle point involves the definition of $\tan \beta$. The angle $\beta$ that appears in the expression for $m_{h^{0}}^{2}$ in eq. (A.5) is implicitly defined at the scale $m_{Z}$. However, for $m_{A^{0}} \gg m_{Z}$, the Higgs sector is effectively the one-doublet scalar sector of the Standard Model below the scale $m_{A^{0}}$. Thus, another choice is to define $\tan \beta$ as the ratio of vacuum expectation values evaluated at $m_{A^{0}}$. To convert between definitions is a simple task. In the expression for $m_{h^{0}}^{2}$ given in eq. (A.5), it suffices to replace the tree-level contribution, $m_{Z}^{2} \cos ^{2} 2 \beta\left(m_{Z}\right)$, using the leading $\log$ expression [16]

$$
\cos ^{2} 2 \beta\left(m_{Z}\right)=\cos ^{2} 2 \beta\left(m_{A^{0}}\right)+\frac{g^{2} N_{c} \cos 2 \beta}{8 \pi^{2} m_{W}^{2}}\left[m_{t}^{2} \cos ^{2} \beta \ln \left(\frac{m_{A^{0}}^{2}}{m_{t}^{2}}\right)-m_{b}^{2} \sin ^{2} \beta \ln \left(\frac{m_{A^{0}}^{2}}{m_{Z}^{2}}\right)\right] .
$$

However, we alert the reader that we have not made this replacement in any of our formulae. If not explicitly indicated, all quoted formulae in this paper assume that $\tan \beta$ is defined at $m_{Z}$.

We can improve the above formulae by reinterpreting the meaning of $M_{\text {SUSY }}$. For example, all terms proportional to $\ln \left(M_{\mathrm{SUSY}}^{2} / m_{t}^{2}\right)$ arise from diagrams with loops involving the top quark and topsquarks. Explicit diagrammatic computations then show that we can reinterpret $M_{\mathrm{SUSY}}^{2}=M_{\tilde{t}_{L}} M_{\tilde{t}_{R}}$. Note that with this reinterpretation of $M_{\mathrm{SUSY}}^{2}$, the top quark and top squark loop contributions to the Higgs masses cancel exactly when $M_{\tilde{t}_{L}}=M_{\tilde{t}_{R}}=m_{t}$, as required in the supersymmetric limit. Likewise, in terms proportional to $P_{b}$ or powers of $m_{b}$ multiplied by $\ln \left(M_{\mathrm{SUSY}}^{2} / m_{Z}^{2}\right)$, we may reinterpret $M_{\mathrm{SUSY}}=M_{\tilde{b}_{L}} M_{\tilde{b}_{R}}$. Terms proportional to $P_{f} \ln \left(M_{\mathrm{SUSY}}^{2} / m_{Z}^{2}\right)$ come from loops of lighter quarks and leptons (and their supersymmetric partners) in an obvious way, and the corresponding $M_{\text {SUSY }}^{2}$ can be reinterpreted accordingly. Additional contributions arising in the case of non-degenerate squark

\footnotetext{
${ }^{n}$ In producing the graphs shown in Sections 2 and 3, we also have omitted the non-leading logarithmic term proportional to $m_{t}^{2}$ that appears in $\Delta \mathcal{M}_{22}^{2}$, since this term is not picked up by the numerical integration of the RGEs.

${ }^{o}$ Note that the above arguments imply that if $m_{A^{0}}=M_{\mathrm{SUSY}}$ then the leading $m_{t}^{4}$ contribution to the $\mathcal{O}\left(g^{2} m_{Z}^{2}\right)$ terms is absent, while for $m_{t} \leq m_{A^{0}} \leq M_{\mathrm{SUSY}}$, the leading $m_{t}^{4}$ term is proportional to $m_{t}^{4} \ln \left(M_{\mathrm{SUSY}}^{2} / m_{A^{0}}^{2}\right)$.
} 
masses are treated in Appendix C. The remaining leading logarithmic terms arise from gauge and Higgs boson loops and their supersymmetric partners. The best we can do in the above formulae is to interpret $M_{\mathrm{SUSY}}$ as an average neutralino and chargino mass. To incorporate thresholds more precisely requires a more complicated version of eq. (A.2), which can be easily derived from formulae given in ref. [16]. The corresponding expressions are summarized in Appendix D. However, the impact of these corrections are no more important than the non-leading logarithmic terms which have been discarded. The most significant non-logarithmic correction is one term that scales with $m_{t}^{2}$ and thus has been included in eq. (A.2). This is surely the largest of such corrections. One can check that it yields at most a $1 \mathrm{GeV}$ shift in the computed Higgs masses.

Finally, we give the one-loop leading logarithmic expression for the charged Higgs mass. First, assuming that $m_{A^{0}} \lesssim \mathcal{O}\left(m_{Z}\right)$,

$$
\begin{aligned}
\left(m_{H^{ \pm}}^{2}\right)_{1 \mathrm{LL}}=m_{A^{0}}^{2}+m_{W}^{2}+ & \frac{N_{c} g^{2}}{32 \pi^{2} m_{W}^{2}}\left[\frac{2 m_{t}^{2} m_{b}^{2}}{\sin ^{2} \beta \cos ^{2} \beta}-m_{W}^{2}\left(\frac{m_{t}^{2}}{\sin ^{2} \beta}+\frac{m_{b}^{2}}{\cos ^{2} \beta}\right)+\frac{2}{3} m_{W}^{4}\right] \ln \left(\frac{M_{\mathrm{SUSY}}^{2}}{m_{t}^{2}}\right) \\
& +\frac{g^{2} m_{W}^{2}}{48 \pi^{2}}\left[N_{c}\left(N_{g}-1\right)+N_{g}-9+15 \tan ^{2} \theta_{W}\right] \ln \left(\frac{M_{\mathrm{SUSY}}^{2}}{m_{W}^{2}}\right) .
\end{aligned}
$$

For $m_{A^{0}} \gg m_{Z}$, additional terms arise proportional to $\ln \left(m_{A^{0}}^{2} / m_{W}^{2}\right)$. For example, for $m_{A^{0}}=M_{\mathrm{SUSY}}$, the leading logarithmic corrections can be obtained from $m_{H^{ \pm}}^{2}=m_{A^{0}}^{2}+m_{W}^{2}$, where $m_{W}^{2}$ is treated as a running parameter evaluated at $m_{A^{0}}$. Re-expressing $m_{W}\left(m_{A^{0}}\right)$ in terms of the physical $W$ mass yields the correct one-loop leading log corrections for $m_{Z} \leq m_{A^{0}} \leq M_{\mathrm{SUSY}}$. Again, we omit the explicit expressions since these corrections generate only a small relative shift to the heavy charged Higgs mass.

A good approximation to the RG-improved Higgs squared-mass corrections is implemented according to the algorithm of Section 2 by replacing $m_{t}$ and $m_{b}$ in the above formulae by the corresponding running parameters evaluated at $\mu_{t}=\sqrt{m_{t} M_{\mathrm{SUSY}}}$ and $\mu_{b}=\sqrt{m_{Z} M_{\mathrm{SUSY}}}$, respectively.

\section{Appendix B: Leading Squark Mixing Corrections to the MSSM Higgs Masses}

When squark mixing effects are taken into account, the CP-even Higgs squared-mass matrix and $m_{H^{ \pm}}^{2}$ receive additional one-loop corrections beyond those given in Appendix A. We need only consider the effect of mixing among the third-generation squarks (since all such mixing effects are proportional to the corresponding quark mass). In order to keep the formulae simple, we assume that the diagonal elements of the $2 \times 2$ squark squared-mass matrices [eqs. (3.23) and (3.24)] are degenerate and given by $M_{\mathrm{SUSY}}^{2}$. (In Appendix C, we treat the more complex case of non-universal squark squared-masses.) It is convenient to define

$$
\begin{array}{ll}
X_{t} \equiv A_{t}-\mu \cot \beta, & Y_{t} \equiv A_{t}+\mu \tan \beta, \\
X_{b} \equiv A_{b}-\mu \tan \beta, & Y_{b} \equiv A_{b}+\mu \cot \beta .
\end{array}
$$


We assume that the mixing terms $m_{t} X_{t}$ and $m_{b} X_{b}$ are not too large. Then, the elements of the CP-even Higgs squared-mass matrix are given by:

$$
\mathcal{M}^{2}=\mathcal{M}_{1 \mathrm{LL}}^{2}+\Delta \mathcal{M}_{\mathrm{mix}}^{2}
$$

where $\mathcal{M}_{1 \mathrm{LL}}^{2}$ has been given in eqs. (A.1) and (A.2), and

$$
\begin{aligned}
\left(\Delta \mathcal{M}_{11}^{2}\right)_{\operatorname{mix}}= & \frac{g^{2} N_{c}}{32 \pi^{2} m_{W}^{2} M_{\mathrm{SUSY}}^{2}}\left[\frac{4 m_{b}^{4} A_{b} X_{b}}{\cos ^{2} \beta}\left(1-\frac{A_{b} X_{b}}{12 M_{\mathrm{SUSY}}^{2}}\right)-\frac{m_{t}^{4} \mu^{2} X_{t}^{2}}{3 M_{\mathrm{SUSY}}^{2} \sin ^{2} \beta}\right. \\
\left(\Delta \mathcal{M}_{22}^{2}\right)_{\operatorname{mix}}= & \frac{g^{2} N_{c}}{32 \pi^{2} m_{W}^{2} M_{\mathrm{SUSY}}^{2}}\left[\frac{4 m_{t}^{4} A_{t} X_{t}}{\sin ^{2} \beta}\left(1-\frac{A_{t} X_{t}}{12 M_{\mathrm{SUSY}}^{2}}\right)-\frac{m_{b}^{4} \mu^{2} X_{b}^{2}}{3 M_{\mathrm{SUSY}}^{2} \cos ^{2} \beta}\right. \\
\left(\Delta \mathcal{M}_{12}^{2}\right)_{\operatorname{mix}}= & \frac{-g^{2} N_{c}}{64 \pi^{2} m_{W}^{2} M_{\mathrm{SUSY}}^{2}}\left[\frac{4 m_{t}^{4} \mu X_{t}}{\sin ^{2} \beta}\left(1-\frac{A_{t} X_{t}}{6 M_{\mathrm{SUSY}}^{2}}\right)+\frac{4 m_{b}^{4} \mu X_{b}}{\cos ^{2} \beta}\left(1-\frac{A_{b} X_{b}}{6 M_{\mathrm{SUSY}}^{2}}\right)\right. \\
& \left.-m_{Z}^{2} m_{t}^{2} \cot \beta\left[X_{t} Y_{t}+\frac{1}{3}\left(\mu^{2}+A_{t}^{2}\right)\right]-m_{Z}^{2} m_{b}^{2} \tan \beta\left[X_{b} Y_{b}+\frac{1}{3}\left(\mu^{2}+A_{b}^{2}\right)\right]\right] .
\end{aligned}
$$

If $m_{Z} \ll m_{A^{0}} \leq M_{\mathrm{SUSY}}$, then it is again convenient to rotate the neutral scalar Higgs basis as discussed in Appendix A. One then finds that the Higgs squared-masses obtained in eq. (A.5) are shifted by the following expressions

$$
\begin{aligned}
&\left(\Delta m_{h^{0}}^{2}\right)_{\mathrm{mix}}= \frac{g^{2} N_{c}}{16 \pi^{2} m_{W}^{2} M_{\mathrm{SUSY}}^{2}}\left\{2 m_{t}^{4} X_{t}^{2}\left(1-\frac{X_{t}^{2}}{12 M_{\mathrm{SUSY}}^{2}}\right)+2 m_{b}^{4} X_{b}^{2}\left(1-\frac{X_{b}^{2}}{12 M_{\mathrm{SUSY}}^{2}}\right)\right. \\
&\left.+\frac{1}{2} m_{Z}^{2} \cos 2 \beta\left[m_{t}^{2}\left(X_{t}^{2}+\frac{1}{3}\left(A_{t}^{2}-\mu^{2} \cot ^{2} \beta\right)\right)-m_{b}^{2}\left(X_{b}^{2}+\frac{1}{3}\left(A_{b}^{2}-\mu^{2} \tan ^{2} \beta\right)\right)\right]\right\} \\
&\left(\Delta m_{H^{0}}^{2}\right)_{\mathrm{mix}}=\frac{g^{2} N_{c}}{16 \pi^{2} m_{W}^{2} M_{\mathrm{SUSY}}^{2}}\left\{2 m_{t}^{4} X_{t} Y_{t} \cot ^{2} \beta\left(1-\frac{X_{t} Y_{t}}{12 M_{\mathrm{SUSY}}^{2}}\right)+2 m_{b}^{4} X_{b} Y_{b} \tan ^{2} \beta\left(1-\frac{X_{b} Y_{b}}{12 M_{\mathrm{SUSY}}^{2}}\right)\right. \\
&\left.-m_{Z}^{2}\left[m_{t}^{2} \cos ^{2} \beta\left(X_{t} Y_{t}+\frac{1}{3}\left(A_{t}^{2}+\mu^{2}\right)\right)+m_{b}^{2} \sin ^{2} \beta\left(X_{b} Y_{b}+\frac{1}{3}\left(A_{b}^{2}+\mu^{2}\right)\right)\right]\right\}
\end{aligned}
$$

Squark mixing effects also lead to modifications of the charged Higgs squared-mass. One finds that the charged Higgs squared-mass obtained in eq. (A.7) is shifted by

$$
\begin{aligned}
\left(m_{H^{ \pm}}^{2}\right)_{\text {mix }}= & \frac{N_{c} g^{2}}{192 \pi^{2} m_{W}^{2} M_{\mathrm{SUSY}}^{2}}\left[\frac{2 m_{t}^{2} m_{W}^{2}\left(\mu^{2}-2 A_{t}^{2}\right)}{\sin ^{2} \beta}+\frac{2 m_{b}^{2} m_{W}^{2}\left(\mu^{2}-2 A_{b}^{2}\right)}{\cos ^{2} \beta}\right. \\
& \left.-3 \mu^{2}\left(\frac{m_{t}^{2}}{\sin ^{2} \beta}+\frac{m_{b}^{2}}{\cos ^{2} \beta}\right)^{2}+\frac{m_{t}^{2} m_{b}^{2}}{\sin ^{2} \beta \cos ^{2} \beta}\left(3\left(A_{t}+A_{b}\right)^{2}-\frac{\left(A_{t} A_{b}-\mu^{2}\right)^{2}}{M_{\mathrm{SUSY}}^{2}}\right)\right] .
\end{aligned}
$$

\footnotetext{
${ }^{p}$ Formally, the expressions given in this Appendix are the results of an expansion in the variable $\left(M_{1}^{2}-M_{2}^{2}\right) /\left(M_{1}^{2}+M_{2}^{2}\right)$, where $M_{1}^{2}, M_{2}^{2}$ are the squared-mass eigenvalues of the squark mass matrix. Thus, we demand that $m_{t} X_{t} / M_{\mathrm{SUSY}}^{2} \ll 1$. For example, for $M_{\mathrm{SUSY}}=1 \mathrm{TeV}$, values of $X_{t} / M_{\mathrm{SUSY}} \lesssim 3$ should yield an acceptable approximation based on the formulae presented here.
} 
A good approximation to the RG-improved Higgs squared-mass corrections is implemented according to the algorithm of Section 3 by replacing $m_{t}$ and $m_{b}$ in the above formulae by the corresponding running parameters evaluated at $\mu=M_{\text {SUSY }}$.

\section{Appendix C: Non-Universal Squark Mass Corrections}

The formulae of Appendices A and B were obtained under the assumption that $M_{Q}=M_{U}=M_{D} \equiv$ $M_{\text {SUSY }}$, where $M_{Q}, M_{U}$, and $M_{D}$ are the diagonal soft-supersymmetry-breaking squark mass parameters defined in eqs. (3.23) and (3.24). In this Appendix, we generalize the results of the previous two appendices to allow for unequal diagonal scalar masses.

First, the results of Appendix A are modified as follows. For the neutral CP-even squared-mass matrix, replace in eqs. (A.2) A.5) all occurrences of $\ln \left(M_{\mathrm{SUSY}}^{2} / m_{t}^{2}\right)$ with $\ln \left(M_{Q} M_{U} / m_{t}^{2}\right)$, and replace all occurrences of $\ln \left(M_{\mathrm{SUSY}}^{2} / m_{Z}^{2}\right)$ which multiply either $m_{b}^{4}, m_{b}^{2}$ or $P_{b}$ by $\ln \left(M_{Q} M_{D} / m_{Z}^{2}\right)$. In addition, eq. (A.2) must be modified by adding the following squared-mass shifts:

$$
\begin{aligned}
& \Delta \mathcal{M}_{11}^{2}=\frac{-g^{2} N_{c} m_{Z}^{2} \cos ^{2} \beta}{32 \pi^{2} \cos ^{2} \theta_{W}}[\left(1+4 e_{b} \sin ^{2} \theta_{W}\right)\left(\frac{m_{b}^{2}}{m_{Z}^{2} \cos ^{2} \beta}-\frac{1}{6}\right) \ln \left(\frac{M_{Q}^{2}}{M_{D}^{2}}\right) \\
&\left.-\frac{1}{6}\left(1-4 e_{t} \sin ^{2} \theta_{W}\right) \ln \left(\frac{M_{Q}^{2}}{M_{U}^{2}}\right)\right], \\
& \Delta \mathcal{M}_{22}^{2}=\frac{-g^{2} N_{c} m_{Z}^{2} \sin ^{2} \beta}{32 \pi^{2} \cos ^{2} \theta_{W}}\left[\left(1-4 e_{t} \sin ^{2} \theta_{W}\right)\left(\frac{m_{t}^{2}}{m_{Z}^{2} \sin ^{2} \beta}-\frac{1}{6}\right) \ln \left(\frac{M_{Q}^{2}}{M_{U}^{2}}\right)\right.\left.-\frac{1}{6}\left(1+4 e_{b} \sin ^{2} \theta_{W}\right) \ln \left(\frac{M_{Q}^{2}}{M_{D}^{2}}\right)\right], \\
& \mathcal{M}_{12}^{2}=\frac{g^{2} N_{c} m_{Z}^{2} \sin \beta \cos \beta}{64 \pi^{2} \cos ^{2} \theta_{W}}\left[\left(1-4 e_{t} \sin ^{2} \theta_{W}\right)\left(\frac{m_{t}^{2}}{m_{Z}^{2} \sin ^{2} \beta}-\frac{1}{3}\right) \ln \left(\frac{M_{Q}^{2}}{M_{U}^{2}}\right)\right. \\
&\left.+\left(1+4 e_{b} \sin ^{2} \theta_{W}\right)\left(\frac{m_{b}^{2}}{m_{Z}^{2} \cos ^{2} \beta}-\frac{1}{3}\right) \ln \left(\frac{M_{Q}^{2}}{M_{D}^{2}}\right)\right] .
\end{aligned}
$$

In the limit of $m_{A^{0}} \gg m_{Z}$ [using eq. (A.4)], we find that the following squared-mass shift must be added to $\left(m_{h^{0}}^{2}\right)_{1 L L}$ given in eq. (A.5):

$$
\begin{aligned}
\Delta m_{h^{0}}^{2}=\frac{g^{2} N_{c} m_{Z}^{2} \cos 2 \beta}{32 \pi^{2} \cos ^{2} \theta_{W}}[ & \left(1-4 e_{t} \sin ^{2} \theta_{W}\right)\left(\frac{m_{t}^{2}}{m_{Z}^{2}}+\frac{1}{6} \cos 2 \beta\right) \ln \left(\frac{M_{Q}^{2}}{M_{U}^{2}}\right) \\
& \left.-\left(1+4 e_{b} \sin ^{2} \theta_{W}\right)\left(\frac{m_{b}^{2}}{m_{Z}^{2}}-\frac{1}{6} \cos 2 \beta\right) \ln \left(\frac{M_{Q}^{2}}{M_{D}^{2}}\right)\right] .
\end{aligned}
$$

For $m_{H^{ \pm}}$, replace $\ln \left(M_{\mathrm{SUSY}}^{2} / m_{t}^{2}\right)$ in eq. (A.7) with $\left.\left.\frac{1}{2}\left[\ln \left(M_{Q} M_{U}\right) / m_{t}^{2}\right)+\ln \left(M_{Q} M_{D}\right) / m_{t}^{2}\right)\right]$. In addition, the following squared-mass shift must be added to $\left(m_{H^{ \pm}}^{2}\right)_{1 \mathrm{LL}}$ given in eq. (A.7):

$$
\begin{aligned}
\Delta m_{H^{ \pm}}^{2}= & \frac{-g^{2} N_{c}}{64 \pi^{2} m_{W}^{2}}\left\{\frac{m_{t}^{2} m_{b}^{2}}{\sin ^{2} \beta \cos ^{2} \beta}\left(M_{U}^{2}-M_{D}^{2}\right)^{2} g\left(M_{U}^{2}, M_{D}^{2}\right)\right. \\
& \left.+\frac{1}{2}\left[m_{W}^{2}\left(\frac{m_{t}^{2}}{\sin ^{2} \beta}+\frac{m_{b}^{2}}{\cos ^{2} \beta}\right)-\frac{2}{3} m_{W}^{4}\right]\left[\ln \left(\frac{M_{Q}^{2}}{M_{U}^{2}}\right)+\ln \left(\frac{M_{Q}^{2}}{M_{D}^{2}}\right)\right]\right\},
\end{aligned}
$$


where the function $g(a, b)$ is defined by:

$$
g(a, b) \equiv \frac{1}{(a-b)^{2}}\left[2-\frac{a+b}{a-b} \ln \left(\frac{a}{b}\right)\right] .
$$

Note that $g(a, a)=-1 / 6 a^{2}$, so that in the limit of $M_{Q}=M_{U}=M_{D}$, all mass shifts given above vanish.

Next, we consider the modifications of the results of Appendix B when the diagonal softsupersymmetry-breaking squark mass parameters are non-degenerate. The shifts in the squared-mass matrix elements of the CP-even Higgs boson given in eq. (B.3) are replaced by the following expressions:

$$
\begin{aligned}
\left(\Delta \mathcal{M}_{11}^{2}\right)_{\text {mix }}=\frac{g^{2} N_{c}}{16 \pi^{2} m_{W}^{2}} & \left\{\frac{m_{b}^{4} A_{b} X_{b}}{\cos ^{2} \beta}\left[2 h\left(M_{Q}^{2}, M_{D}^{2}\right)+A_{b} X_{b} g\left(M_{Q}^{2}, M_{D}^{2}\right)\right]+\frac{m_{t}^{4} \mu^{2} X_{t}^{2}}{\sin ^{2} \beta} g\left(M_{Q}^{2}, M_{U}^{2}\right)\right. \\
& +m_{Z}^{2} m_{t}^{2} \mu \cot \beta\left[X_{t} p_{t}\left(M_{Q}^{2}, M_{U}^{2}\right)-\mu \cot \beta B\left(M_{Q}^{2}, M_{U}^{2}\right)\right] \\
& \left.+m_{Z}^{2} m_{b}^{2} A_{b}\left[X_{b} p_{b}\left(M_{Q}^{2}, M_{D}^{2}\right)-A_{b} B\left(M_{Q}^{2}, M_{D}^{2}\right)\right]\right\}, \\
\left(\Delta \mathcal{M}_{22}^{2}\right)_{\text {mix }}=\frac{g^{2} N_{c}}{16 \pi^{2} m_{W}^{2}} & \left\{\frac{m_{t}^{4} A_{t} X_{t}}{\sin ^{2} \beta}\left[2 h\left(M_{Q}^{2}, M_{U}^{2}\right)+A_{t} X_{t} g\left(M_{Q}^{2}, M_{U}^{2}\right)\right]+\frac{m_{b}^{4} \mu^{2} X_{b}^{2}}{\cos ^{2} \beta} g\left(M_{Q}^{2}, M_{D}^{2}\right)\right. \\
& +m_{Z}^{2} m_{b}^{2} \mu \tan \beta\left[X_{b} p_{b}\left(M_{Q}^{2}, M_{D}^{2}\right)-\mu \tan \beta B\left(M_{Q}^{2}, M_{D}^{2}\right)\right] \\
& \left.+m_{Z}^{2} m_{t}^{2} A_{t}\left[X_{t} p_{t}\left(M_{Q}^{2}, M_{U}^{2}\right)-A_{t} B\left(M_{Q}^{2}, M_{U}^{2}\right)\right]\right\}, \\
\left(\Delta \mathcal{M}_{12}^{2}\right)_{\operatorname{mix}}=\frac{-g^{2} N_{c}}{32 \pi^{2} m_{W}^{2}} & \left\{\frac{2 m_{t}^{4}}{\sin ^{2} \beta} \mu X_{t}\left[h\left(M_{Q}^{2}, M_{U}^{2}\right)+A_{t} X_{t} g\left(M_{Q}^{2}, M_{U}^{2}\right)\right]\right. \\
& +\frac{2 m_{b}^{4}}{\cos ^{2} \beta} \mu X_{b}\left[h\left(M_{Q}^{2}, M_{D}^{2}\right)+A_{b} X_{b} g\left(M_{Q}^{2}, M_{D}^{2}\right)\right] \\
& -m_{Z}^{2} m_{b}^{2} \tan \beta\left[\left(\mu^{2}+A_{b}^{2}\right) B\left(M_{Q}^{2}, M_{D}^{2}\right)-X_{b} Y_{b} p_{b}\left(M_{Q}^{2}, M_{D}^{2}\right)\right] \\
& \left.-m_{Z}^{2} m_{t}^{2} \cot \beta\left[\left(\mu^{2}+A_{t}^{2}\right) B\left(M_{Q}^{2}, M_{U}^{2}\right)-X_{t} Y_{t} p_{t}\left(M_{Q}^{2}, M_{U}^{2}\right)\right]\right\},
\end{aligned}
$$

where the functions $B, h, p_{b}$ and $p_{t}$ are defined as follows:

$$
\begin{aligned}
h(a, b) & =\frac{1}{a-b} \ln \left(\frac{a}{b}\right), \\
B(a, b) & =\frac{1}{(a-b)^{2}}\left[\frac{1}{2}(a+b)-\frac{a b}{a-b} \ln \left(\frac{a}{b}\right)\right], \\
p_{b}(a, b) & =f(a, b)-2 e_{b} \sin ^{2} \theta_{W}(a-b) g(a, b), \\
p_{t}(a, b) & =f(a, b)+2 e_{t} \sin ^{2} \theta_{W}(a-b) g(a, b),
\end{aligned}
$$

$g(a, b)$ is given in eq. (C.4) and $f(a, b)$ is given by:

$$
f(a, b)=\frac{-1}{a-b}\left[1-\frac{b}{a-b} \ln \left(\frac{a}{b}\right)\right] .
$$

The contributions to $\Delta \mathcal{M}_{\text {mix }}^{2}$ proportional to $m_{b}^{4}$ or $m_{t}^{4}$ were first given in Ref. [8]. 
To check that eq. (C.5) reduces to eq. (B.3) in the limit of $M_{Q}=M_{U}=M_{D}$, one makes use of $B(a, a)=1 / 6 a, f(a, a)=-1 / 2 a, g(a, a)=-1 / 6 a^{2}$, and $h(a, a)=1 / a$. In the limit of $m_{A^{0}} \gg m_{Z}$, eq. (B.4) is replaced by:

$$
\begin{aligned}
\left(\Delta m_{h^{0}}^{2}\right)_{\text {mix }}=\frac{g^{2} N_{c}}{16 \pi^{2} m_{W}^{2}} & \left\{m_{t}^{4} X_{t}^{2}\left[2 h\left(M_{Q}^{2}, M_{U}^{2}\right)+X_{t}^{2} g\left(M_{Q}^{2}, M_{U}^{2}\right)\right]\right. \\
& m_{b}^{4} X_{b}^{2}\left[2 h\left(M_{Q}^{2}, M_{D}^{2}\right)+X_{b}^{2} g\left(M_{Q}^{2}, M_{D}^{2}\right)\right] \\
+ & m_{Z}^{2} m_{t}^{2} \cos 2 \beta\left[\left(A_{t}^{2}-\mu^{2} \cot ^{2} \beta\right) B\left(M_{Q}^{2}, M_{U}^{2}\right)-X_{t}^{2} p_{t}\left(M_{Q}^{2}, M_{U}^{2}\right)\right] \\
& \left.-m_{Z}^{2} m_{b}^{2} \cos 2 \beta\left[\left(A_{b}^{2}-\mu^{2} \tan ^{2} \beta\right) B\left(M_{Q}^{2}, M_{D}^{2}\right)-X_{b}^{2} p_{b}\left(M_{Q}^{2}, M_{D}^{2}\right)\right]\right\}, \\
\left(\Delta m_{H^{0}}^{2}\right)_{\operatorname{mix}}=\frac{g^{2} N_{c}}{16 \pi^{2} m_{W}^{2}} & \left\{m_{t}^{4} X_{t} Y_{t} \cot ^{2} \beta\left[2 h\left(M_{Q}^{2}, M_{U}^{2}\right)+X_{t} Y_{t} g\left(M_{Q}^{2}, M_{U}^{2}\right)\right]\right. \\
& m_{b}^{4} X_{b} Y_{b} \tan ^{2} \beta\left[2 h\left(M_{Q}^{2}, M_{D}^{2}\right)+X_{b} Y_{b} g\left(M_{Q}^{2}, M_{D}^{2}\right)\right] \\
+ & 2 m_{Z}^{2} m_{t}^{2} \cos ^{2} \beta\left[X_{t} Y_{t} p_{t}\left(M_{Q}^{2}, M_{U}^{2}\right)-\left(\mu^{2}+A_{t}^{2}\right) B\left(M_{Q}^{2}, M_{U}^{2}\right)\right] \\
+ & \left.2 m_{Z}^{2} m_{b}^{2} \sin ^{2} \beta\left[X_{b} Y_{b} p_{b}\left(M_{Q}^{2}, M_{D}^{2}\right)-\left(\mu^{2}+A_{b}^{2}\right) B\left(M_{Q}^{2}, M_{D}^{2}\right)\right]\right\} .
\end{aligned}
$$

The shift in the charged Higgs squared-mass given in eq. (B.5) is replaced by:

$$
\begin{aligned}
\left(m_{H^{ \pm}}^{2}\right)_{\text {mix }}=\frac{g^{2} N_{c}}{32 \pi^{2} m_{W}^{2}}\left\{\mu^{2}\left[\frac{m_{t}^{4}}{\sin ^{2} \beta} f\left(M_{Q}^{2}, M_{U}^{2}\right)+\frac{m_{b}^{4}}{\cos ^{2} \beta} f\left(M_{Q}^{2}, M_{D}^{2}\right)+\frac{2 m_{t}^{2} m_{b}^{2}}{\sin ^{2} \beta \cos ^{2} \beta} F\left(M_{Q}^{2}, M_{U}^{2}, M_{D}^{2}\right)\right]\right. \\
-\frac{m_{b}^{2} m_{t}^{2}}{\sin ^{2} \beta \cos ^{2} \beta}\left[A_{b}^{2} f\left(M_{Q}^{2}, M_{D}^{2}\right)+A_{t}^{2} f\left(M_{Q}^{2}, M_{U}^{2}\right)+2 A_{b} A_{t} F\left(M_{Q}^{2}, M_{U}^{2}, M_{D}^{2}\right)\right. \\
\left.+\left(\mu^{2}-A_{t} A_{b}\right)^{2} G\left(M_{Q}^{2}, M_{U}^{2}, M_{D}^{2}\right)\right] \\
-\frac{m_{W}^{2} m_{t}^{2}}{\sin ^{2} \beta}\left(\mu^{2}\left[f\left(M_{Q}^{2}, M_{U}^{2}\right)+b\left(M_{Q}^{2}, M_{U}^{2}\right)\right]-A_{t}^{2}\left[f\left(M_{Q}^{2}, M_{U}^{2}\right)-B\left(M_{Q}^{2}, M_{U}^{2}\right)\right]\right) \\
\left.-\frac{m_{W}^{2} m_{b}^{2}}{\cos ^{2} \beta}\left(\mu^{2}\left[f\left(M_{Q}^{2}, M_{D}^{2}\right)+b\left(M_{Q}^{2}, M_{D}^{2}\right)\right]-A_{b}^{2}\left[f\left(M_{Q}^{2}, M_{D}^{2}\right)-B\left(M_{Q}^{2}, M_{D}^{2}\right)\right]\right)\right\},
\end{aligned}
$$

where the new functions that appear depend on three variables:

$$
\begin{aligned}
G(a, b, c) & =\frac{f(a, b)-f(a, c)}{b-c}, \\
F(a, b, c) & =\frac{(b-a) f(a, b)-(c-a) f(a, c)}{b-c} .
\end{aligned}
$$

To check that eq. (C.9) reduces to eq. (B.5) in the limit of $M_{Q}=M_{U}=M_{D}$, one should note that $F(a, c, c)=f(c, a)$ and $G(a, c, c)=-g(a, c)$. To take the limit of $a=c$, we may use the results quoted below eq. (C.7).

The above results correspond to the leading terms of an expansion in $\left(M_{1}^{2}-M_{2}^{2}\right) /\left(M_{1}^{2}+M_{2}^{2}\right)$, where $M_{1}^{2}, M_{2}^{2}$ are the squared-mass eigenvalues of the squark mass matrix. If the squark squared-mass splitting is large, one needs a slightly better result. This is easily obtained from the formulae above for the CP-even Higgs squared-masses as follows. Terms multiplying factors of $m_{b}$ involve functions 
of the arguments $M_{Q}^{2}$ and $M_{D}^{2}$. In these terms, replace $M_{Q}^{2}$ and $M_{D}^{2}$ by the corresponding bottom squark squared-masses. Similarly, terms multiplying factors of $m_{t}$ involve functions of the arguments $M_{Q}^{2}$ and $M_{U}^{2}$. In these terms, replace $M_{Q}^{2}$ and $M_{U}^{2}$ by the corresponding top squark squared-masses. One can check that this rule correctly reproduces the terms in the CP-even Higgs squared-mass matrix proportional to $m_{t}^{4}$ and $m_{b}^{4}$ obtained by the effective potential methods of Ref. [8]. Moreover, we have explicitly verified that the terms proportional to $m_{t}^{2}$ are also correctly reproduced at $\beta=\pi / 2$ by comparing with the exact one-loop computation of Ref. [5]. In the case of the charged Higgs mass, the improvement required in the case of large squark squared-mass splitting is more complicated. In particular, the simple rule quoted above does not apply, since there are functions appearing in the charged Higgs mass formulae that contain both $M_{U}^{2}$ and $M_{D}^{2}$ as arguments, so the effects of top and bottom squarks do not factorize.

Finally, we must address the question of effective scales $\mu_{q}$ and $\mu_{\tilde{q}}(q=t, b)$ introduced in Sections 2 and 3, respectively, to account for the renormalization group improvement. By iteratively solving the RGEs with two different squark masses we can show that the two-loop leading log corrections can be absorbed into the one-loop expression by choosing

$$
\ln \frac{\mu_{t}^{2}}{m_{t}^{2}}=\frac{\ln ^{2}\left(M_{\tilde{t}_{1}} / m_{t}\right)+\ln ^{2}\left(M_{\tilde{t}_{2}} / m_{t}\right)}{\ln \left(M_{\tilde{t}_{1}} M_{\tilde{t}_{2}} / m_{t}^{2}\right)},
$$

and similarly for $\mu_{b}$ [with $m_{t}$ replaced by $m_{Z}$ in the arguments of the logarithms]. Similarly, one can modify the analysis at the end of Section 3 to show that the $\mu_{\tilde{q}}$ defined below eq. (3.25) generalizes to $\mu_{\tilde{q}} \equiv M_{\tilde{q}_{2}}(q=t, b)$, i.e., the mass of the corresponding heaviest squark mass eigenvalue.

To illustrate the effects of the corrections described in this section, we exhibit in Fig. 10 the radiatively corrected RG-improved light CP-even Higgs mass as function of $M_{\mathrm{SUSY}}$ for $m_{A^{0}}=A_{t}=$ $-\mu=M_{Q}=M_{U}=M_{D}=M_{\mathrm{SUSY}}$ and two choices of $\tan \beta=1.5$ and $\tan \beta=20$. The graphs have been computed using the algorithm of eq. (3.25). The dotted line is based on the formulae presented in Appendix $\mathrm{A}$ and $\mathrm{B}$, where terms proportional to $\ln \left(m_{A^{0}}^{2} / m_{Z}^{2}\right)$ and the non-leading logarithmic term proportional to $m_{t}^{2}$ have been omitted, as described below eq. (A.5). The importance of these two terms is illustrated by including them in the dashed curve of Fig. 10. This curve also includes the effect of replacing $M_{\text {SUSY }}^{2}$ in the leading logs of Appendix A by the appropriate product of third generation squark masses, as described below eq. A.6). We call this the "improved one-scale" approximation. Finally, we improve on this approximation by employing the correction terms described in this section, with the replacement of $M_{Q}, M_{U}$, and $M_{D}$ by the corresponding third generation squark masses as described below eq. (C.11). The result of this improved treatment of third generation squark thresholds is depicted by the solid line in Fig. 10. One can see that the improved treatment of thresholds typically introduces no more than a $2 \mathrm{GeV}$ mass shift in the predicted value of $m_{h^{0}}$.

It is important to emphasize that all the formulae given in Appendices $\mathrm{B}$ and $\mathrm{C}$ are based on the assumption that the top and bottom squark masses are substantially larger than $m_{Z}$. Fortunately, in most cases of interest, the error introduced if the squark masses are not sufficiently heavy is rather small, of the same size as the non-logarithmic terms that have been systematically neglected in this paper. Thus, one can confidently use the formulae given in this paper over most of the relevant supersymmetric parameter space, and expect an accuracy in the computed Higgs masses within $\sim 2 \mathrm{GeV}$ of their actual values. 


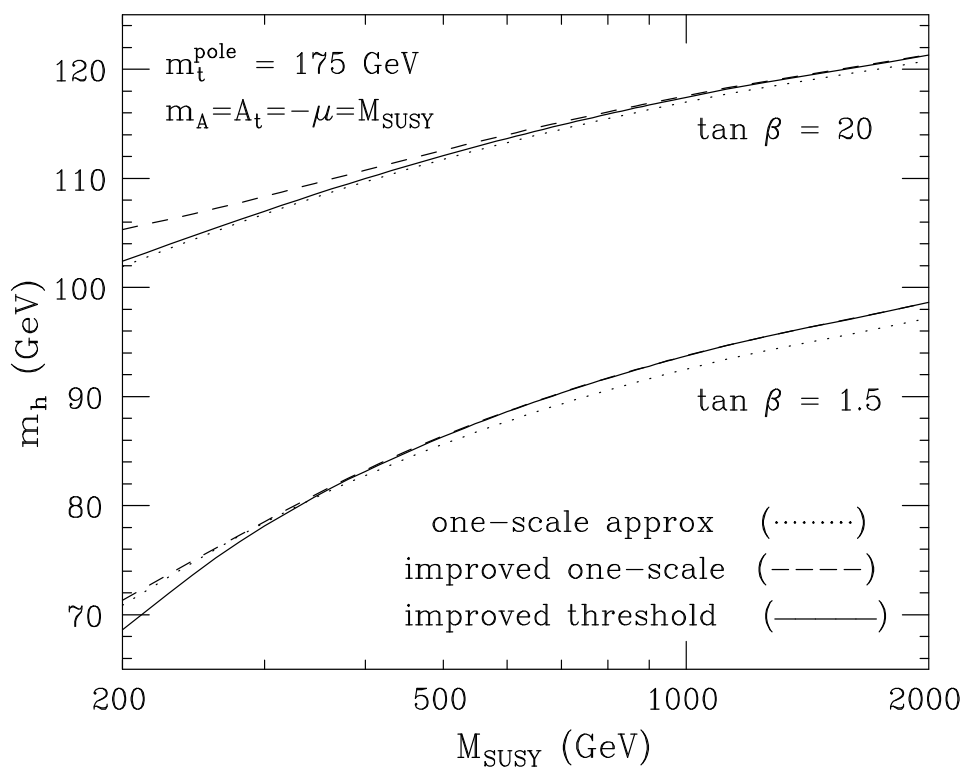

Fig. 10. The radiatively corrected light RG-improved CP-even Higgs mass is plotted as a function of $M_{\mathrm{SUSY}}$ for $m_{A^{0}}=A_{t}=-\mu=M_{Q}=M_{U}=M_{D}=M_{\mathrm{SUSY}}$. Two choices of $\tan \beta=1.5$ and $\tan \beta=20$ are shown. The results are based on the analytic approximation given in eq. (3.25). In the one-scale approximation (dotted line), the distinction between $M_{\text {SUSY }}$ and third generation squark masses are neglected. In addition, radiative corrections proportional to $\ln \left(m_{A^{0}}^{2} / m_{Z}^{2}\right)$ and the non-leading $\log$ term proportional to $m_{t}^{2}$ are neglected, as discussed below eq. A.5 ). These approximations were also made in the graphs of all previous figures. In the improved one-scale approximation (dashed line), the latter two neglected terms are included. In addition, the factors of $M_{\text {SUSY }}^{2}$ in the argument of the leading logs of Appendix $\mathrm{A}$ are replaced by the appropriate product of third generation squark masses, as described below eq. (A.6). In the improved threshold approximation, we include the corrections of Appendix C with the replacement of $M_{Q}, M_{U}$, and $M_{D}$ by the corresponding third generation squark masses as described below eq. (C.11). 


\section{Appendix D: Non-universal Gaugino and Higgsino Mass Corrections}

The formulae of Appendix A were obtained under the assumption that the chargino and neutralino masses are all degenerate and equal to $M_{\text {SUSY }}$ (assumed large compared to $m_{Z}$ ). In this appendix, we relax the assumption of degeneracy of masses, although we still assume that all the chargino and neutralino masses are large compared to $m_{Z}$. If the latter condition is not satisfied, then the logarithmic pieces that we keep are of the same order of magnitude as the non-logarithmic pieces that we omit. Nevertheless, the contributions of the charginos and neutralinos to the Higgs masses are small (never exceeding $2 \mathrm{GeV}$ ), so our approximations will be accurate at this level over the entire neutralino and chargino parameter space. The results below have been extracted from ref. 16].

In the limit where all chargino and neutralino masses are large compared to $m_{Z}$, the chargino spectrum consists of two states of mass $M_{2}$ and $|\mu|$ respectively, while the neutralino spectrum consists of two states of mass $M_{1}, M_{2}$, and two approximately degenerate states of mass $|\mu|$. Here, $M_{1}$ and $M_{2}$ are Majorana masses for the uncolored gauginos, and $\mu$ is the supersymmetric Higgs mass parameter

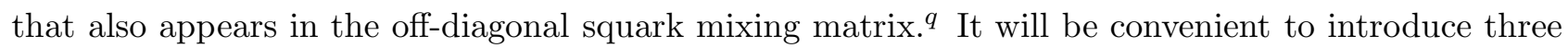
mass parameters which are equal to either $M_{1}, M_{2}$, or $|\mu|$ depending on their relative magnitude:

$$
\begin{aligned}
& \mu_{1} \equiv \max \left\{|\mu|, M_{1}\right\} \\
& \mu_{2} \equiv \max \left\{|\mu|, M_{2}\right\} \\
& \mu_{12} \equiv \max \left\{|\mu|, M_{1}, M_{2}\right\}
\end{aligned}
$$

We consider the case of unequal $M_{1}, M_{2}$, and $|\mu|$, all of which are assumed to be large compared to $m_{Z}$. Then, eq. (A.2) of Appendix A is modified by adding the following squared-mass shifts:

$$
\begin{aligned}
\Delta \mathcal{M}_{11}^{2}= & \frac{g^{2} m_{Z}^{2} \cos ^{2} \beta}{96 \pi^{2} \cos ^{2} \theta_{W}}\left\{6 \sin ^{2} \theta_{W}\left(1-2 \sin ^{2} \theta_{W}\right) \ln \left(\frac{\mu_{1}^{2}}{m_{Z}^{2}}\right)-24 \sin ^{2} \theta_{W} \cos ^{2} \theta_{W} \ln \left(\frac{\mu_{12}^{2}}{m_{Z}^{2}}\right)\right. \\
& +6 \cos ^{2} \theta_{W}\left(3-10 \cos ^{2} \theta_{W}\right) \ln \left(\frac{\mu_{2}^{2}}{m_{Z}^{2}}\right)-8 \cos ^{4} \theta_{W} \ln \left(\frac{M_{2}^{2}}{m_{Z}^{2}}\right) \\
& \left.-4\left(\sin ^{4} \theta_{W}+\cos ^{4} \theta_{W}\right) \ln \left(\frac{\mu^{2}}{m_{Z}^{2}}\right)-\left(P_{g}+P_{2 H}\right) \ln \left(\frac{M_{\mathrm{SUSY}}^{2}}{m_{Z}^{2}}\right)\right\}, \\
\mathcal{M}_{22}^{2}= & \frac{g^{2} m_{Z}^{2} \sin ^{2} \beta}{96 \pi^{2} \cos ^{2} \theta_{W}}\left\{6 \sin ^{2} \theta_{W}\left(1-2 \sin ^{2} \theta_{W}\right) \ln \left(\frac{\mu_{1}^{2}}{m_{Z}^{2}}\right)-24 \sin ^{2} \theta_{W} \cos ^{2} \theta_{W} \ln \left(\frac{\mu_{12}^{2}}{m_{Z}^{2}}\right)\right. \\
& +6 \cos ^{2} \theta_{W}\left(3-10 \cos ^{2} \theta_{W}\right) \ln \left(\frac{\mu_{2}^{2}}{m_{Z}^{2}}\right)-8 \cos ^{4} \theta_{W} \ln \left(\frac{M_{2}^{2}}{m_{Z}^{2}}\right) \\
& \left.-4\left(\sin ^{4} \theta_{W}+\cos ^{4} \theta_{W}\right) \ln \left(\frac{\mu^{2}}{m_{Z}^{2}}\right)-\left(P_{g}+P_{2 H}\right) \ln \left(\frac{M_{\mathrm{SUSY}}^{2}}{m_{Z}^{2}}\right)\right\}, \\
& -g^{2} m_{Z}^{2} \sin ^{2} \cos \beta \\
\hline \mathcal{M}_{12}^{2}=6 \pi^{2} \cos ^{2} \theta_{W} & +6 \cos ^{2} \theta_{W}\left(3+2 \theta_{W}\left(1+2 \sin ^{2} \theta_{W}\right) \ln \left(\frac{\mu_{1}^{2}}{m_{Z}^{2}}\right)+24 \sin ^{2} \theta_{W} \cos ^{2} \theta_{W} \ln \left(\frac{\mu_{2}^{2}}{m_{Z}^{2}}\right)-8 \cos ^{4} \theta_{W} \ln \left(\frac{M_{2}^{2}}{m_{Z}^{2}}\right)\right. \\
& \left.-4\left(\sin ^{4} \theta_{W}+\cos ^{4} \theta_{W}\right) \ln \left(\frac{\mu^{2}}{m_{Z}^{2}}\right)-\left(P_{g}^{\prime}+P_{2 H}^{\prime}\right) \ln \left(\frac{M_{\mathrm{SUSY}}^{2}}{m_{Z}^{2}}\right)\right\},
\end{aligned}
$$

${ }^{q}$ We follow ref. 27] for the definitions of the MSSM parameters. 
where the $P_{i}$ are defined in eq. (A.3). In the limit of $m_{A^{0}} \gg m_{Z}$ [using eq. (A.4)], we find that the following squared-mass shift must be added to $\left(m_{h^{0}}^{2}\right)_{1 \mathrm{LL}}$ given in eq. (A.5):

$$
\begin{aligned}
\Delta m_{h^{0}}^{2}= & \frac{g^{2} m_{Z}^{2}}{96 \pi^{2} \cos ^{2} \theta_{W}}\left\{6 \sin ^{2} \theta_{W}\left(\cos ^{2} 2 \beta-2 \sin ^{2} \theta_{W}\right) \ln \left(\frac{\mu_{1}^{2}}{m_{Z}^{2}}\right)-24 \sin ^{2} \theta_{W} \cos ^{2} \theta_{W} \ln \left(\frac{\mu_{12}^{2}}{m_{Z}^{2}}\right)\right. \\
& -6 \cos ^{2} \theta_{W}\left[\cos ^{2} 2 \beta\left(1-4 \sin ^{2} \theta_{W}\right)+6 \cos ^{2} \theta_{W}\right] \ln \left(\frac{\mu_{2}^{2}}{m_{Z}^{2}}\right)-8 \cos ^{4} \theta_{W} \cos ^{2} 2 \beta \ln \left(\frac{M_{2}^{2}}{m_{Z}^{2}}\right) \\
& -4\left(\cos ^{4} \theta_{W}+\sin ^{4} \theta_{W}\right) \cos ^{2} 2 \beta \ln \left(\frac{\mu^{2}}{m_{Z}^{2}}\right) \\
& \left.-\left[\left(P_{g}+P_{2 H}\right)\left(\sin ^{4} \beta+\cos ^{4} \beta\right)-2\left(P_{g}^{\prime}+P_{2 H}^{\prime}\right) \sin ^{2} \beta \cos ^{2} \beta\right] \ln \left(\frac{M_{\text {SUSY }}^{2}}{m_{Z}^{2}}\right)\right\} .
\end{aligned}
$$

Note that the effect of the terms proportional to $\ln \left(M_{\mathrm{SUSY}}^{2} / m_{Z}^{2}\right)$ is to simply remove the $M_{\mathrm{SUSY}}$ dependence in eqs. (A.2) and (A.4) that arises from the gauge/Higgs/gaugino/higgsino contributions. These contributions are now more accurately described by logarithmic factors that are sensitive to the gaugino/higgsino spectrum.

For $m_{H^{ \pm}}^{2}$, the following squared-mass shift must be added to $\left(m_{H^{ \pm}}^{2}\right)_{1 \mathrm{LL}}$ given in eq. (A.7):

$$
\begin{aligned}
\Delta m_{H^{ \pm}}^{2}= & \frac{-g^{2} m_{W}^{2}}{48 \pi^{2}}\left[3 \tan ^{2} \theta_{W} \ln \left(\frac{\mu_{1}^{2}}{m_{W}^{2}}\right)+12 \tan ^{2} \theta_{W} \ln \left(\frac{\mu_{12}^{2}}{m_{W}^{2}}\right)-3 \ln \left(\frac{\mu_{2}^{2}}{m_{W}^{2}}\right)\right. \\
& \left.-4 \ln \left(\frac{M_{2}^{2}}{m_{W}^{2}}\right)-2 \ln \left(\frac{\mu^{2}}{m_{W}^{2}}\right)-\left(15 \tan ^{2} \theta_{W}-9\right) \ln \left(\frac{M_{\text {SUSY }}^{2}}{m_{W}^{2}}\right)\right] .
\end{aligned}
$$

As above, the effect of the terms proportional to $\ln \left(M_{\mathrm{SUSY}}^{2} / m_{W}^{2}\right)$ is to simply remove the $M_{\mathrm{SUSY}}$ dependence in eq. (A.7) that arises from the gauge/Higgs/gaugino/higgsino contributions.

One can easily check that if $M_{1}=M_{2}=|\mu|=M_{\mathrm{SUSY}}$, then all the mass shifts in this appendix vanish. In the case of the neutral [charged] Higgs mass computation, if any of the gaugino/higgsino mass parameters is less than $m_{Z}\left[m_{W}\right]$, then one must remove the corresponding logarithmic term from the above expressions. Note that the supersymmetric limit corresponds to all three gaugino/higgsino mass parameters zero and $\tan \beta=1$, in which case two charginos are degenerate with the $W^{ \pm}$and $H^{ \pm}$, two neutralinos are degenerate with the $Z$ and $H^{0}$, one neutralino is degenerate with the photon, and one neutralino is degenerate with the (massless) $h^{0}$ and $A^{0}$. In this case, we must remove all logarithmic terms above, except for the log terms containing $M_{\text {SUSY }}^{2}$. The effect of the latter is to precisely cancel the contributions of the gauge and Higgs loops to the CP-even Higgs squared-mass matrix [eqs. (A.2) and (A.4)] and the charged Higgs mass [eq. (A.7)]. This cancellation is a consequence of unbroken supersymmetry. Of course, as emphasized above, when some of the gaugino/higgsino mass parameters are of order $m_{Z}$, then the above corrections terms are small in magnitude and of the same order as non-logarithmic corrections not included in this analysis. 


\section{References}

1. G. Altarelli, T. Sjostrand and F. Zwirner (editors), Proceedings of the Workshop on Physics at LEP2, CERN Yellow Report 96-01 (1996).

2. See e.g. N. Cabibbo, L. Maiani, G. Parisi and R. Petronzio, Nucl. Phys. B158 (1979) 295; M. Lindner, Z. Phys. C31 (1986) 295.

3. For a recent analysis, see G. Altarelli and G. Isidori, Phys. Lett. B337 (1994) 141; J.R. Espinosa and M. Quiros, Phys. Lett. B353 (1995) 257.

4. For a comprehensive review of the MSSM Higgs sector, see chapter 4 of J.F. Gunion, H.E. Haber, G.L. Kane and S. Dawson, The Higgs Hunter's Guide (Addison-Wesley, Redwood City, CA, 1990).

5. H.E. Haber and R. Hempfling, Phys. Rev. Lett. 66 (1991) 1815.

6. Y. Okada, M. Yamaguchi and T. Yanagida, Prog. Theor. Phys. 85 (1991) 1; J. Ellis, G. Ridolfi and F. Zwirner, Phys. Lett. B257 (1991) 83.

7. S.P. Li, M. Sher, Phys. Lett. B140 (1984) 339; A. Brignole, J. Ellis, G. Ridolfi and F. Zwirner, Phys. Lett. B271 (1991) 123 [E: B273 (1991) 550]; M. Drees and M.M. Nojiri, Phys. Rev. D45 (1992) 2482; Phys. Rev. D46 (3086) 1992; J.A. Casas, J.R. Espinosa, M. Quiros, A. Riotto, Nucl. Phys. B436 (1995) 3 [E: B439 (1995) 466].

8. J. Ellis, G. Ridolfi and F. Zwirner, Phys. Lett. B262 (1991) 477.

9. M. Carena, J.R. Espinosa, M. Quiros, and C.E.M. Wagner, Phys. Lett. B355 (1995) 209; M. Carena, M. Quiros, and C.E.M. Wagner, Nucl. Phys. B461 (1996) 407.

10. J.F. Gunion and A. Turski, Phys. Rev. D39 (1989) 2701; Phys. Rev. D40 (1989) 2333; M.S. Berger, Phys. Rev. D41 (1990) 225; A. Brignole, Phys. Lett. B277 (1992) 313; M.A. Díaz and H.E. Haber, Phys. Rev. D46 (3086) 1992.

11. M.A. Díaz and H.E. Haber, Phys. Rev. D45 (4246) 1992.

12. R. Hempfling and A.H. Hoang, Phys. Lett. B331 (1994) 99.

13. P.H. Chankowski, S. Pokorski, J. Rosiek, Phys. Lett. B274 (1992) 191; Nucl. Phys. B423 (1994) 437; A. Brignole, Phys. Lett. B281 (1992) 284; A. Yamada, Phys. Lett. B263 (1991) 233; Z. Phys. C61 (1994) 247; A. Dabelstein, Z. Phys. C67 (1995) 495; D.M. Pierce, J.A. Bagger, K. Matchev and R. Zhang, SLAC-PUB-7180 hep-ph/9606211 (1996).

14. R. Barbieri, M. Frigeni and F. Caravaglios, Phys. Lett. B258 (1991) 167; Y. Okada, M. Yamaguchi, T. Yanagida, Phys. Lett. B262 (1991) 54; D.M. Pierce, A. Papadopoulos and S. Johnson, Phys. Rev. Lett. 68 (1992) 3678. K. Sasaki, M. Carena, C.E.M. Wagner, Nucl. Phys. B381 (1992) 66; R. Hempfling, in Phenomenological Aspects of Supersymmetry, edited by W. Hollik, R. Rückl, and J. Wess (Springer-Verlag, Berlin, $1992)$ p. 260; J. Kodaira, Y. Yasui, K. Sasaki, Phys. Rev. D50 (1994) 7035.

15. J.R. Espinosa and M. Quiros, Phys. Lett. B266 (1991) 389.

16. H.E. Haber and R. Hempfling, Phys. Rev. D48 (1993) 4280. 
17. M. Carena, P. Zerwas et al., "Higgs Physics," in Report of the Higgs Physics Working Group of the LEP-2 Workshop, in Proceedings of the Workshop on Physics at LEP2, Vol. 1, G. Altarelli, T. Sjostrand and F. Zwirner (editors), CERN Yellow Report 96-01 (1996) p. 351.

18. H.E. Haber, "Recent Refinements in Higgs Physics", in the Proceedings of the 1995 International Europhysics Conference on High Energy Physics, 27 July-2 August, 1995, Brussels, Belgium, edited by J. Lemonne, C. Vander Velde, and F. Verbeure (World Scientific, Singapore, 1996) p. 15.

19. J.F. Gunion, A. Stange, and S. Willenbrock, "Weakly Coupled Higgs Bosons", UCD-95-28 (1995), to appear in Electroweak Symmetry Breaking and New Physics at the TeV Scale, edited by T.L. Barklow, S. Dawson, H.E. Haber, and J.L. Siegrist (World Scientific, Singapore, 1996).

20. We use the formulae quoted in Appendix A of Ref. [16]. Further citations to the original literature can be found there.

21. J.-F. Grivaz, "New Particle Searches", in the Proceedings of the 1995 International Europhysics Conference on High Energy Physics, 27 July-2 August, 1995, Brussels, Belgium, edited by J. Lemonne, C. Vander Velde, and F. Verbeure (World Scientific, Singapore, 1996) p. 827.

22. R.M. Barnett et al. [Particle Data Group], Phys. Rev. D54 (1996) 1.

23. R. Tarrach, Nucl. Phys. B183 (1981) 384; N. Gray, D.J. Broadhurst, W. Grafe, and K. Schilcher, Z. Phys. C48 (1990) 673.

24. R. Hempfling and B.A. Kniehl, Phys. Rev. D51 (1995) 1386.

25. A. Sirlin and R. Zucchini, Nucl. Phys. B266 (1986) 389;

26. I. Jack and H. Osborn, J. Phys. A16 (1983) 1101; M.E. Machacek and M.T. Vaughn, Nucl. Phys. B249 (1985) 70; C. Ford, I. Jack, and D.R.T. Jones, Nucl. Phys. B387 (1992) 373. For a useful compilation of two-loop RGEs for the Standard Model and the MSSM, see B. Schrempp and M. Wimmer, Prog. Part. Nucl. Phys. 37 (1996) 1.

27. H.E. Haber, Phys. Rev. D54 (1996) 687.

28. For a recent study of color and electric charge breaking minima in the MSSM and references to earlier works, see J.A. Casas, A. Lleyda and C. Muñoz, Nucl. Phys. B471 (1996) 3 . 